\title{
Notas y comentarios
}

\section{Análisis del consumo de información en la revista Estudios Demográficos y Urbanos, 1998-2007*}

\author{
Eduardo Ruvalcaba Burgoa**
}

Uno de los métodos utilizados para identificar los patrones en el consumo de información que siguen los investigadores de un área determinada es el análisis de citas. El objetivo del presente documento es determinar mediante dicho método los hábitos informativos que siguen los autores que han publicado en la revista Estudios Demográficos y Urbanos, grupo integrado principalmente por especialistas en demografia, estudios urbanos y ambientales. Con ello se busca incidir en el desarrollo de las colecciones de la Biblioteca Daniel Cosío Villegas de El Colegio de México en dichas temáticas.

Se analizaron 6559 citas bibliográficas de 162 artículos publicados en la revista durante el periodo 1998-2007. La información se registró en una base de datos preparada en Microsoft Access y posteriormente se exportó a una hoja de cálculo en Microsoft Excel, con la finalidad de analizar cuantitativamente los datos con base en indicadores que fueron previamente definidos.

Entre los resultados a destacar se encuentran los siguientes: los libros cubren el mayor porcentaje de las obras citadas (34.7\%); México es el país en el que se edita la mayoría de los documentos citados (38.92\%); Estudios Demográficos y Urbanos es la revista más citada por los investigadores que publicaron durante ese periodo; el espanol $(51.55 \%)$ y el inglés (43.33\%) son los idiomas más consultados por los autores. Sólo $26.73 \%$ del total de las referencias citadas fueron publicadas en los cinco años previos a la fecha en que fueron consultadas, lo que puede deberse a la escasa literatura sobre temas nuevos, enfoques innovadores y materias muy especializadas; a la dificultad para obtener información actualizada y confiable tanto de México como de Latinoamérica y, en ciertos casos, a la poca actualidad del material que los autores consultan, entre otros factores.

El análisis de los resultados obtenidos nos permite realizar algunas sugerencias respecto a las políticas de desarrollo de las colecciones, las cuales están relacionadas con el idioma y la actualidad de los documentos. Asimismo nos muestra la necesidad de reforzar la adquisición de colecciones como las del INEGI y el Conapo.

* El invaluable apoyo y la guía metodológica de la doctora Inés Aragón González, profesora titular del Departamento de Biblioteconomía y Documentación de la Universidad Carlos III de Madrid, han sido elemento fundamental para la culminación de este documento.

** Biblioteca Daniel Cosío Villegas de El Colegio de México y Universidad Carlos III de Madrid. Correo electrónico: eruvalcaba@colmex.mx. 
Palabras clave: consumo de información, análisis de citas, políticas de desarrollo de colecciones.

Analysis of Use of Information in Estudios Demográficos y Urbanos, 1998-2007

One of the methods used to identify patterns in the use of information consulted by researchers in a set area is the analysis of quotes. The aim of this article is to use this method to determine the information habits of authors that have published in the Estudios Demográficos y Urbanos journal, a group mainly comprising specialists in demography, urban and environmental studies. The aim is to influence the development of the collections in the Daniel Cosí Villegas Library at El Colegio de México on these topics.

A total of 6,559 bibliographical quotes from 162 articles published in the journal during the period from 1998/2007 were analyzed. The information was registered in a database prepared by Microsoft Access and subsequently exported to a Microsoft Excel spreadsheet, in order to quantitatively analyze the data on the basis of previously defined indicators.

The most outstanding results are given below: Books account for the highest percentage of works cited (34.7\%); Mexico is the country where the majority of the documents cited are edited (38.92\%); Estudios Demográficos y Urbanos is the journal most widely cited by the researchers that published during that period; Spanish (51.55\%) and English (43.33\%) are the languages most frequently consulted by the authors. Only $26.73 \%$ of the total references cited were published in the five years prior to the date in which they were consulted, which may be due to the lack of literature on new topics, innovative approaches and highly specialized subjects, the difficulty of obtaining reliable, up-to-date information on either Mexico or Latin America and in some cases, to the out-of-date material consulted by the authors, among other factors.

An analysis of the results obtained enabled the author to make suggestions about the policies for developing the collections, related to the language they are published in and the topicality of the documents. It also shows the need to increase the acquisition of collections such as those in INEGI and Conapo.

Key words: use of information, analysis of quotes, policies for developing collections.

\section{Introducción}

La literatura bibliotecológica mexicana registra pocos estudios en los que se ha analizado el consumo de información de los investigadores en las áreas de ciencias sociales con base en las citas que han empleado en sus trabajos de investigación. Una búsqueda realizada en Información y Bibliotecología Latinoamericana (Infobila), la base de datos más importante de la especialidad en la región, muestra la escasez de este 
tipo de estudios no sólo en México sino en toda América Latina. De ahí que surja el interés por realizar un análisis de los patrones de consumo de información en una de las revistas académicas más importantes en el área de las ciencias sociales.

Son dos las razones fundamentales que justifican la selección de la revista Estudios Demográficos y Urbanos (EDU) para realizar este análisis: en primer lugar, el vínculo académico laboral que me une con el Centro que edita la revista; en segundo lugar, el interés por investigar el consumo de la información que realizan los especialistas en demografía, estudios urbanos y estudios ambientales, teniendo como base una importante revista de América Latina.

\section{Estudios Demográficos y Urbanos}

EDU es publicada cuatrimestralmente por el Centro de Estudios Demográficos, Urbanos y Ambientales (CEDUA) de El Colegio de México, institución pública de enseñanza superior e investigación. Surge con la idea de servir como medio de difusión para los resultados de la investigación que se realiza en el CEDUA; sin embargo, como sucede con la mayoría de las revistas académicas, es también un canal de comunicación entre los investigadores pertenecientes a otras instituciones nacionales y extranjeras que trabajan temas afines. $E D U$ publica artículos exclusivamente en español, por lo que si un documento escrito en otro idioma es dictaminado favorablemente, será traducido. Cabe aclarar que la revista incluye una traducción al inglés del resumen y las palabras clave de cada artículo publicado.

Para que un artículo sea publicado en EDU es requisito que cuente con al menos dos dictámenes favorables. Éstos son elaborados por especialistas en el tema a revisar, y se realizan bajo el formato de "doble ciego" (el autor desconoce quiénes evalúan su trabajo, y los dictaminadores ignoran quién es el autor del texto).

Debido a la calidad de sus contenidos, a su sólido comité editorial y a la rigurosidad de sus dictámenes, la revista forma parte del Índice de Revistas Mexicanas de Investigación Científica y Tecnológica del Consejo Nacional de Ciencia y Tecnología (Conacyt). Asimismo se encuentra indizada en las fuentes más importantes de su especialidad como son: Population Index, Popline y Sociological Abstracts, además de otras de carácter general como Scopus, Citas Latinoamericanas en Ciencias Sociales y Humanidades (CLASE), Dialnet y el Hispanic Ame- 
rican Periodical Index (HAPI). EDU cubre actualmente 30 de los 33 criterios de calidad editorial definidos por el Sistema Regional de Información en Línea para Revistas Científicas de América Latina, el Caribe, España y Portugal (Latindex), por lo que está incluido tanto en su directorio como en su catálogo. La revista, que se publica en formato impreso, cuenta también con una versión en línea con acceso gratuito al texto completo a través del sitio de las revistas electrónicas de El Colegio de México (se exceptúan los números publicados en el último año, que El Colegio de México embarga para promover las suscripciones en su versión en papel). Asimismo EDU forma parte del acervo de la Red de Revistas Científicas de América Latina y el Caribe, España y Portugal (Redalyc), cuyos contenidos pueden consultarse en línea, también de manera gratuita.

\section{Objetivos del estudio}

El objetivo de este estudio es investigar, con base en el análisis de citas, los patrones de consumo de la información que siguen los investigadores que han publicado en $E D U$ durante el periodo 1998-2007. Asimismo se pretende ofrecer posibles pautas para el desarrollo de las colecciones en las áreas de demografía, estudios urbanos y medio ambiente de la Biblioteca Daniel Cosío Villegas de El Colegio de México.

\section{Revisión de la literatura}

El método de análisis de citas es apropiado para el estudio de los patrones de consumo de información en la medida que ofrece datos más exactos acerca de las fuentes que consultan los investigadores, en este caso, de quienes publicaron en $E D U$ durante el periodo referido. En una revisión bibliográfica realizada sobre el tema, Urbano Salido (2001) analizó las contribuciones de la citación, y la asumió como indicador de uso. Tal y como afirma el autor en una de sus conclusiones, "conocer qué usan realmente los investigadores de una determinada área del conocimiento en una universidad puede ser un dato de gran interés en el proceso de volver a definir las políticas de adquisiciones a la vista del nuevo entorno digital".

La aplicación del análisis de citas como una herramienta para el desarrollo de las colecciones en las bibliotecas no es un tema novedoso. 
Los enfoques que se han brindado son diversos y en distintas áreas. A pesar de ello son pocos los estudios que se han realizado en las ciencias sociales y las humanidades. La mayoría ha estudiado el uso de la información que hacen las comunidades científicas, por lo que algunos resultados son previsibles; por ejemplo, que en dichas comunidades se consulta en mayor medida la colección de publicaciones periódicas que la de monografias. A pesar de lo anterior, puede haber cambios significativos de una comunidad de investigadores a otra, y entre países. Por ello es necesario seguir explorando el análisis de citas como una herramienta para el desarrollo de colecciones ya que su principal fortaleza es que ofrece información con un mayor grado de objetividad.

Algunos especialistas se preguntarán, por ejemplo, por qué no puede una biblioteca basarse en los resultados del Journal Citation Report (JCR) para la selección de su colección de publicaciones periódicas. Estudios como el de Altmann y Gorman (1999) han demostrado que el factor de impacto de una revista -principal indicador del JCR- no puede ser sustituto de los estudios de uso que se realizan de manera local en las bibliotecas. Como ejemplo podemos mencionar que áreas como la historia se verían seriamente afectadas si la selección de títulos en las bibliotecas dependiera de los resultados del JCR, debido a la preferencia que se les brinda a las revistas anglosajonas.

\section{Análisis de citas y desarrollo de colecciones}

Son muchos los estudios que se han realizado para vincular el análisis de citas con el desarrollo de colecciones. Prácticamente la totalidad de éstos está dirigida al análisis de comunidades académicas de profesores, investigadores y estudiantes de posgrado en distintas universidades y centros de investigación. La mayoría de dichos estudios han tenido objetivos muy específicos sobre algún aspecto del desarrollo de colecciones: presupuesto, selección, descarte, políticas de selección. Por ejemplo, la aplicación del análisis de citas para solucionar problemas como el descarte de colecciones o la cancelación de suscripciones fueron parte del estudio realizado por Brown (2002). El resultado principal de ese análisis derivó en la liberación de aproximadamente 243 metros lineales de espacio en la estantería de la University of Michigan Law Library.

Por otra parte, algunos análisis de citas se han enfocado en un aspecto indispensable en el desarrollo de colecciones: el presupuesto. 
Burdick y Sullivan (1993) pudieron determinar así que destinar 88\% del presupuesto a publicaciones periódicas y $12 \%$ a monografías era el parámetro más adecuado para la distribución del presupuesto en las bibliotecas del área de la salud en Estados Unidos y Canadá.

La evaluación de las colecciones también está presente en los documentos que utilizan el análisis de citas como herramienta (Soehner y Richards, 1992; Sylvia, 1998), y algunos emplean metodologías más completas en las que se incluyen el conteo de citas, el análisis de costos por citación y los conteos de intercalación (Sylvia y Lesher, 1995).

El estudio realizado por Aziagba (1993) es uno de los que guarda mayor similitud con el nuestro, ya que su objetivo fue proponer pautas enfocadas a la selección y el desarrollo de una colección de publicaciones periódicas con base en los patrones de consumo de información identificados en la revista del propio centro de investigación.

Finalmente Ashman (2009) muestra que aproximadamente 6.8\% de todos los estudios de análisis de citas recientes de 1995 en adelante, están dirigidos a apoyar las tareas de desarrollo de colecciones.

Estudios basados en los títulos consultados por profesores e investigadores

Muchos de los estudios se basan en los títulos consultados por los investigadores, y ofrecen resultados del poco uso que hacen de determinados títulos de publicaciones periódicas. El beneficio para el desarrollo de las colecciones de una biblioteca es que permite evaluar la posibilidad de cancelar las suscripciones de las revistas poco consultadas. Wesleyan University Library (Klassen, 2001) canceló al menos cien suscripciones después de realizar un estudio entre los profesores, con el que se demostró que un porcentaje considerable de títulos no había sido citado o usado en ningún momento.

Sin embargo no todos los estudios provocan la cancelación de títulos. Por ejemplo, el realizado en la City University of New York's City College (Lascar y Mendelsohn, 2001) dio como resultado la suscripción a seis nuevos títulos de publicaciones periódicas. Por su parte, Walcott (1994) demostró que, contrario a lo que se puede pronosticar, las monografias y las tesis son importantes en las áreas científicas, como las ciencias marinas, conclusión a la que llegó después de haber analizado la producción científica correspondiente a los años 1986-1999 de los profesores del SUNY en el Stony Brook's Marine Science Research Center. 
Por su parte, el estudio realizado por Ovens (1995) tuvo su origen en el recorte de $10 \%$ del presupuesto destinado a la adquisición de publicaciones periódicas, y estuvo basado en el análisis de citas de un grupo de profesores de ciencias, así como en una encuesta. Uno de sus resultados mostró que $43.41 \%$ de los documentos que los profesores citan fueron obtenidos de otros colegas.

Otros estudios como el de McCain (1997) y Nisonger (1998) son muy interesantes en el aspecto teórico, ya que ofrecen un panorama de los conceptos y herramientas vinculados al análisis de citas y su relación con el desarrollo de las colecciones, en particular las de las publicaciones periódicas.

Aunque con una finalidad distinta, los estudios realizados por Pérez Andrés, Estrada Lorenzo, Villar Álvarez y Rebollo Rodríguez (2007) y Aleixandre, Giménez Sánchez, Terrada y López Piñero (1994) han servido como base para plantear la metodología y los indicadores a seguir en el presente estudio.

\section{Material y método}

\section{Material de estudio}

Como fuente principal de información para el estudio se descargaron del sitio en internet de $E D U$ todos los artículos publicados en la revista entre los años 2000 a 2007. Los artículos correspondientes a los años 1998 y 1999 se consultaron en la versión impresa de la revista, disponible en la Biblioteca Daniel Cosío Villegas de El Colegio de México.

$E D U$ publicó durante ese periodo algunos documentos que tipificó como Notas y Comentarios, los cuales no fueron incluidos en este estudio porque su naturaleza es diferente a la de los artículos. Las Notas son textos inéditos que no poseen la profundidad de aquéllos, pero pueden tener un valor metodológico o ilustrativo de casos de aplicación de procesos de investigación; también pueden ser resultado del proceso de dictaminación de textos originalmente presentados para su inclusión en la sección de Artículos, y que a juicio de los dictaminadores no reúnen las características de éstos, pero se considera pertinente difundir su contenido. Por su parte, los Comentarios son reflexiones y opiniones sustentadas respecto de artículos publicados. Cabe destacar que tanto las Notas como los Comentarios están sometidos a 
un dictamen por parte de un miembro del Comité Editorial. De igual manera, fueron excluidos de este estudio los documentos publicados como Reseñas.

\section{Recopilación de datos}

Se extrajeron las citas bibliográficas que aparecen en el apartado de bibliografía al final de cada uno de los artículos publicados en la revista durante el periodo referido. De acuerdo con los indicadores especificados para el estudio, se registró la información en una base de datos creada para tales efectos en el manejador de bases de datos Microsoft Access, que posteriormente se exportó a una hoja de cálculo en Microsoft Excel con la finalidad de analizarla cuantitativamente.

\section{Indicadores}

Para este estudio se plantearon los siguientes indicadores: principales revistas y libros citados por los autores de EDU, país de publicación, idioma, género documental, actualidad de los trabajos, y autores más citados. Con estos indicadores se busca ofrecer respuestas a las siguientes preguntas:

- ¿Cuáles son las principales revistas y libros citados por los autores?

- ¿De qué países se consume más información en estas áreas?

- ¿Cuál es la actualidad de los trabajos que son citados?

- ¿Quiénes son los autores más citados en esas áreas?

- ¿Qué idiomas son los más consultados para la elaboración de sus investigaciones?

- ¿Qué tipo de documentos son los que consultan con mayor frecuencia? 


\section{La información a analizar}

El cuadro 1 muestra la distribución de los 162 artículos analizados correspondientes al periodo 1998-2009. De éstos se obtuvo un total de 6559 citas, que promedian 40.5 citas por artículo. Como puede apreciarse, cada número de la revista publica entre 4 y 7 artículos, algunos de los cuales llegan a tener más de 100 citas.

Se considera que el periodo seleccionado puede ofrecer información confiable y actualizada sobre los patrones de conducta respecto al consumo de información que actualmente siguen los investigadores que han publicado en $E D U$ en esos diez años.

Un aspecto importante a tomar en consideración es que $E D U$ ha sido un medio de comunicación para académicos en los ámbitos nacional e internacional, como se muestra en la gráfica 1, ya que si bien la mayoría de los autores que han publicado en esta revista durante el periodo pertenecen a instituciones mexicanas, se aprecia la contribución de autores procedentes de distintas partes del mundo, lo que en muchos de los casos es producto de la colaboración con académicos nacionales.

Como ya se mencionaba, EDU es uno de los medios más importantes mediante el cual se difunden los resultados de la investigación que llevan a cabo los investigadores del CEDUA. De ahí que casi una quinta parte $(19.05 \%)$ del total de autores que publicaron en la revista durante el periodo analizado procedan del Centro, como se puede apreciar en el cuadro 2. No obstante, las contribuciones de otras instituciones como la Universidad Nacional Autónoma de México y la Universidad Autónoma Metropolitana son muy destacadas. Resalta también la producción de autores estadounidenses y franceses, entre otros de diferentes nacionalidades.

En cuanto a los autores más productivos (cuadro 3), destacan dos investigadores del CEDUA y uno del Instituto Tecnológico y de Estudios Superiores de Monterrey, Campus Ciudad de México, cada uno con cinco contribuciones durante el periodo. Hay que resaltar las frecuentes aportaciones de autores que no pertenecen al CEDUA, pero que encuentran en la revista un canal importante para la difusión de sus resultados de investigación. 


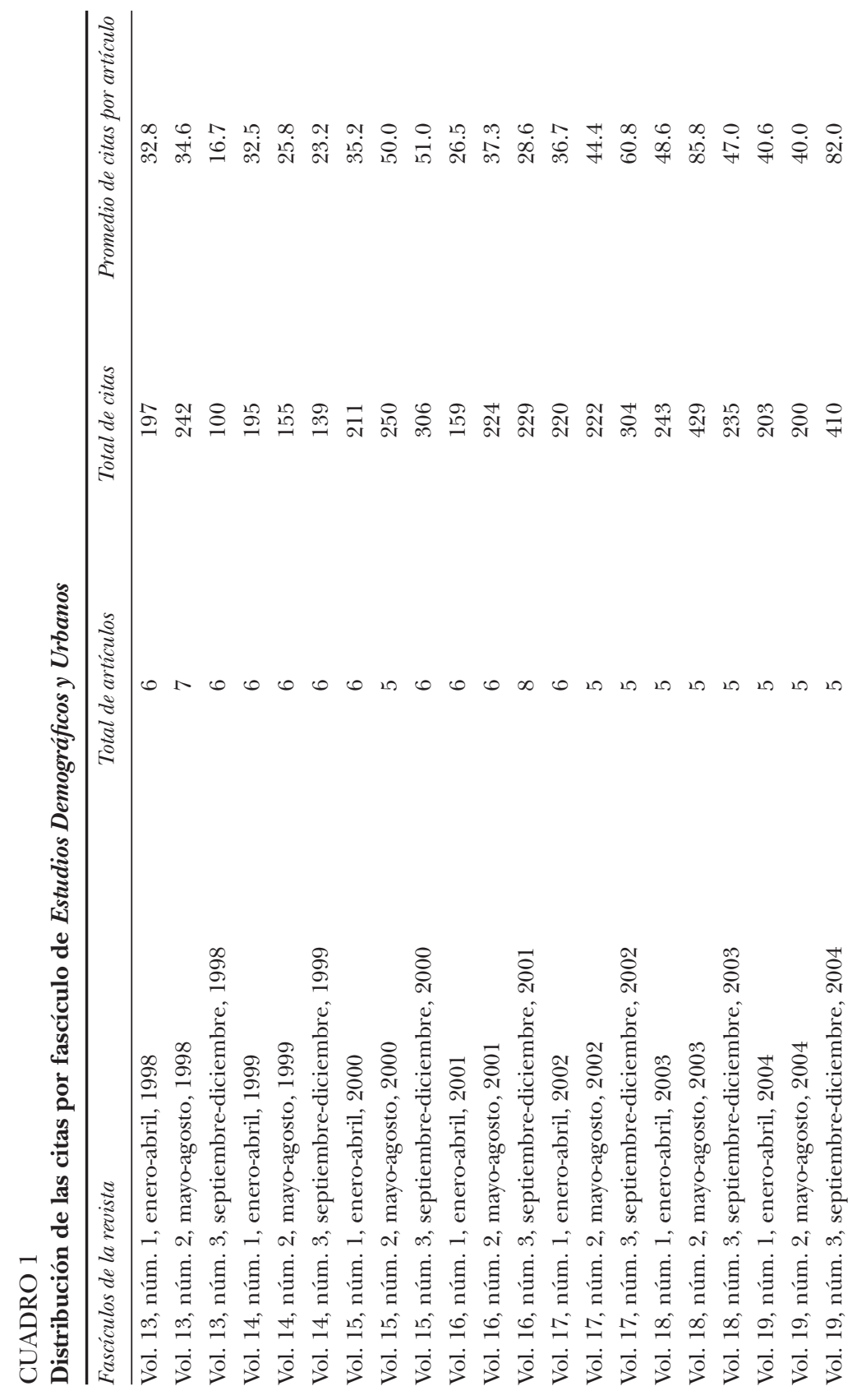




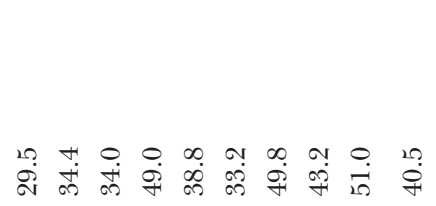

少企。

H $1020+42010+42010 \quad 0$

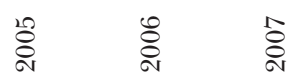

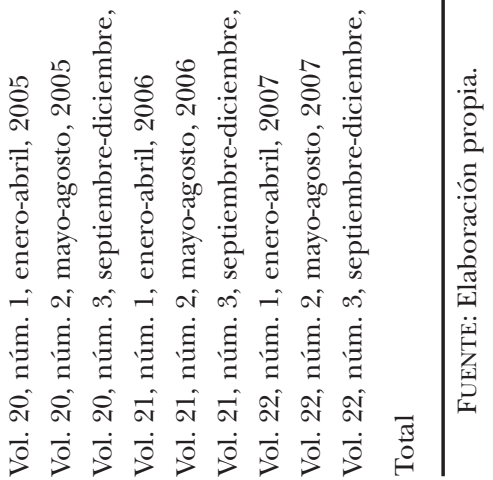




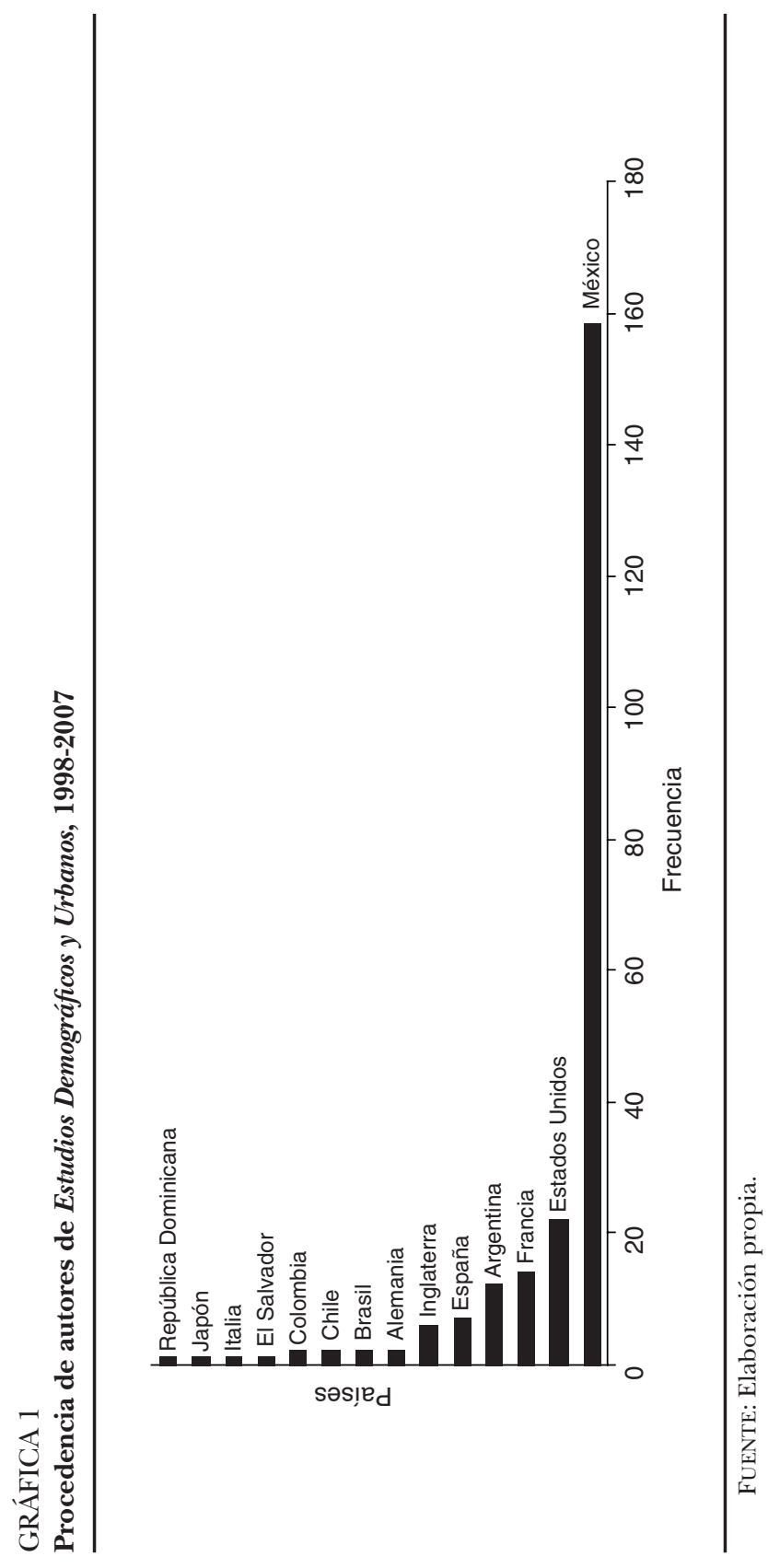




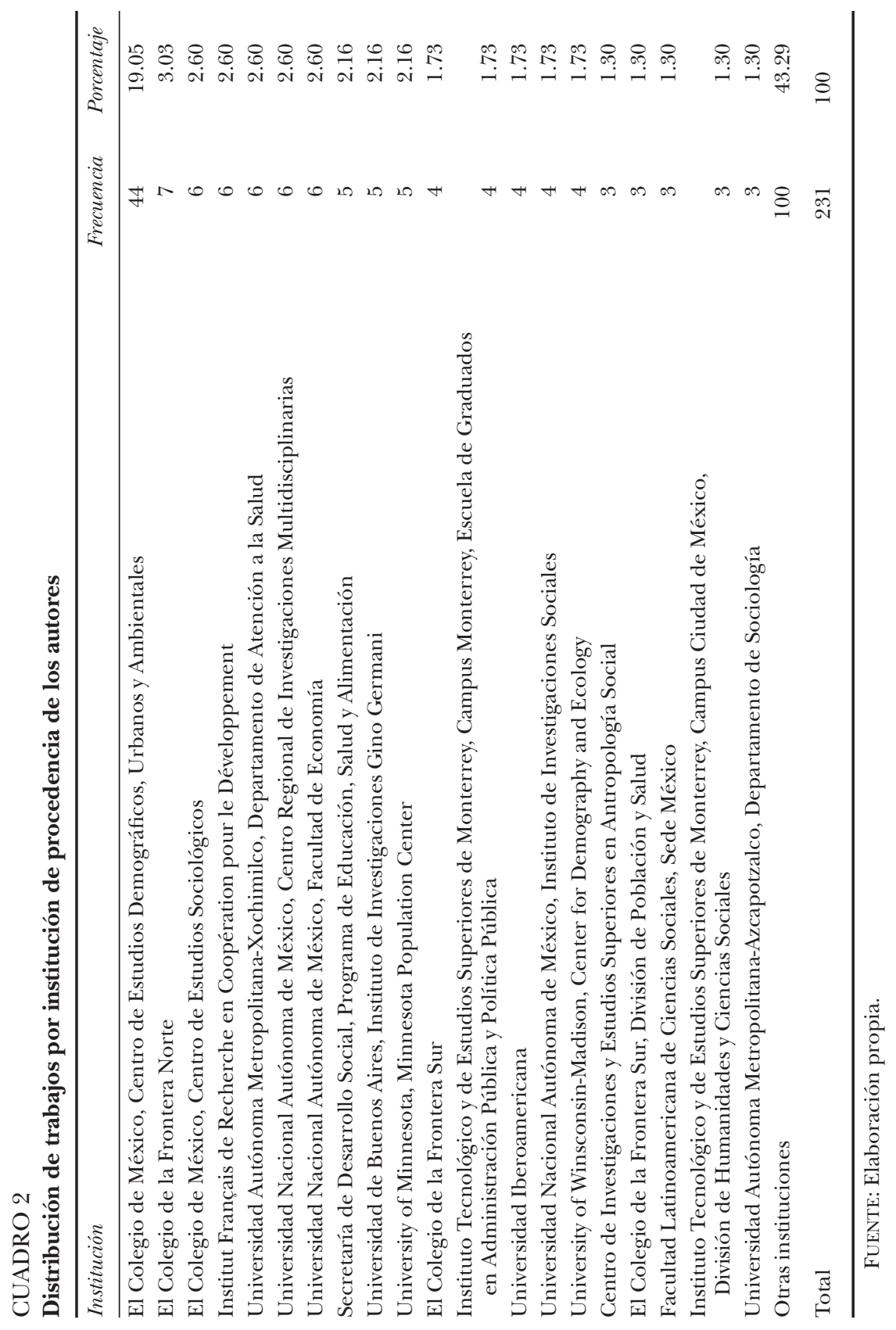


CUADRO 3

Autores más productivos en Estudios Demográficos y Urbanos de 1998 a 2007

\begin{tabular}{clc}
\hline Autor & Institución de procedencia & Contribuciones \\
\hline 1 & CEDUA & 5 \\
2 & CEDUA & 5 \\
3 & ITESM & 5 \\
4 & CEDUA & 4 \\
5 & El Colegio de la Frontera Norte & 3 \\
6 & CES & 3 \\
7 & University of Winsconsin-Madison & 3 \\
8 & Institute de Recherche pour le Développement & 3 \\
9 & CEDUA & 3 \\
10 & CEDUA & 3 \\
11 & ITESM & 3 \\
Otros autores & & 170 \\
Total & & 231 \\
\hline
\end{tabular}

FUENTE: Elaboración propia.

\section{Medición de la colaboración}

Con base en la cantidad de artículos publicados en $E D U$ durante el periodo referido (162) y el número de autores que escribieron en dicho lapso (231), se obtuvo un índice de colaboración de 1.42. ${ }^{1}$ Esta cifra es normal en áreas asociadas a las ciencias sociales, en las que las contribuciones individuales suelen tener mayor peso que las que involucran la participación de dos o más autores.

Como ya se mencionó, uno de los objetivos de este análisis es ofrecer posibles pautas para el desarrollo de colecciones de la Biblioteca Daniel Cosío Villegas de El Colegio de México. Sin embargo debemos tomar en consideración que a pesar de que gran número de los autores de $E D U$ no pertenecen a El Colegio de México y que tampoco son usuarios de la biblioteca, conocer sus hábitos de consumo de información brinda la posibilidad de detectar las posibles deficiencias y fortalezas de las colecciones en las áreas de demografía, urbanismo y medio ambiente. Esto elimina un posible sesgo, en caso de que el

${ }^{1} \mathrm{El}$ índice de colaboración está representado por el número promedio de firmas que han intervenido en la preparación de las publicaciones que son motivo del estudio. 
CUADRO 4

Número de firmantes por trabajo fuente

\begin{tabular}{cc}
\hline Núm. de firmantes & Frecuencia absoluta (núm. de casos) \\
\hline 1 & 110 \\
2 & 43 \\
3 & 4 \\
4 & 3 \\
5 & 1 \\
6 & 1 \\
Total & 162 \\
\hline
\end{tabular}

FUENTE: Elaboración propia.

análisis estuviera dirigido sólo a artículos publicados por investigadores del CEDUA, ya que las colecciones de la Biblioteca son consultadas por los estudiantes de El Colegio de México y por los usuarios externos interesados en los temas de su acervo.

\section{Resultados}

\section{Tipo de documentos}

Al ser EDU una revista del área de las ciencias sociales, podría esperarse un porcentaje considerablemente mayor de citas para libros o capítulos de libros, ya que habitualmente en las ciencias sociales y las humanidades $-\mathrm{y}$ por distintos motivos también en las aplicaciones prácticas- predominan éstas, en tanto que los artículos de las revistas tienen un peso relativamente modesto (Díaz Grau, Mínguez y Osca Lluch, 1999).

Como se observa en el cuadro 5, la consulta de libros entre los investigadores que publicaron en $E D U$ durante el periodo fue ligeramente mayor $(34.7 \%)$ a la de artículos de revistas $(32.7 \%)$. Los capítulos de libros (18.8\%) son también fuente primordial de información en estas áreas, pero lamentablemente no se cuenta con fuentes de información precisas, como las que hay para las publicaciones periódicas. Por ejemplo, no existen bases de datos o índices que den cuenta de la información que se publica en forma de capítulo de libro. Por otra parte, llama la atención la consulta a documentos de literatura gris, los cuales abarcan un porcentaje significativo (5.4\%) de los docu- 


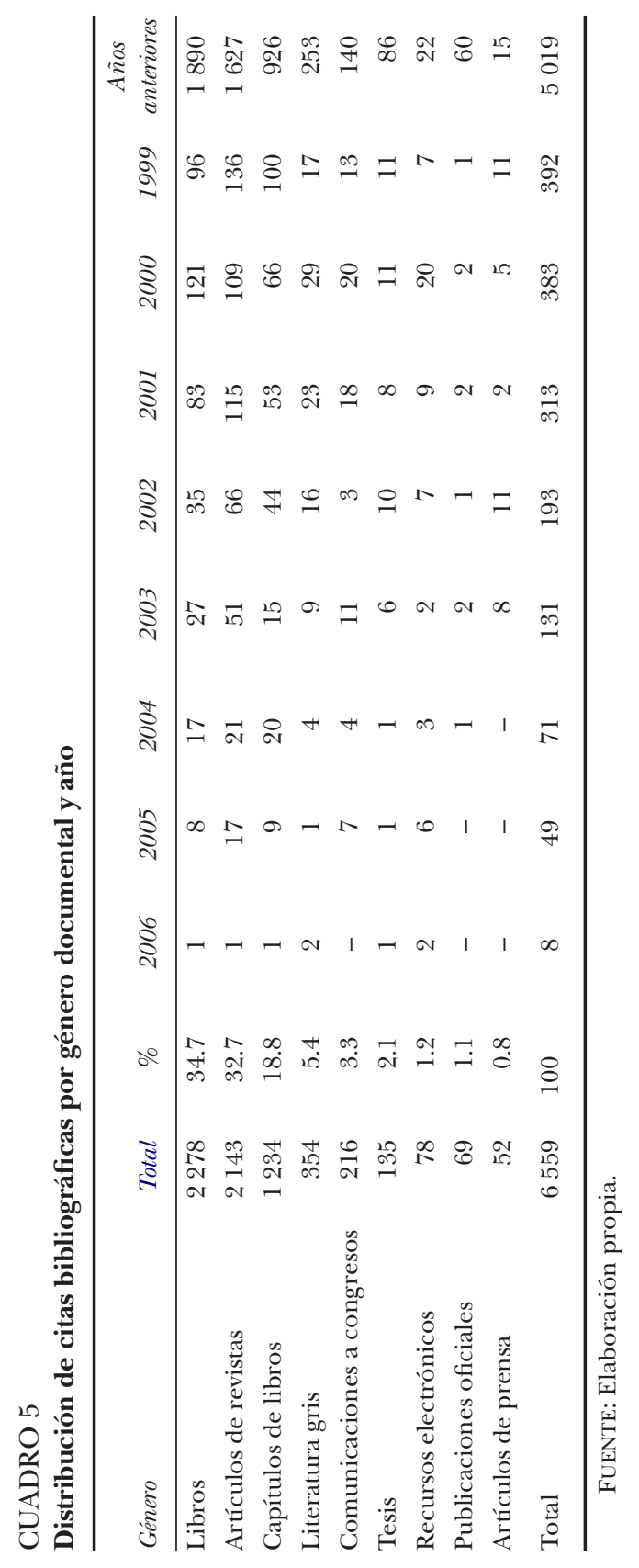


mentos consultados. ${ }^{2}$ Un dato que destaca es el poco uso que se hace de los recursos electrónicos (1.2\%); sin embargo, con base en la información disponible es difícil determinar el formato en el que fue consultada la información. Prácticamente la totalidad de las fuentes han sido citadas como si se tratara de documentos impresos, por lo que no se puede deducir cuántos y cuáles documentos fueron consultados por internet o por otro medio electrónico, ya que una gran cantidad de éstos, principalmente artículos de revistas y literatura gris, son de fácil acceso vía la red.

Los datos anteriores nos muestran la importancia que tienen tanto las monografías como las revistas académicas para los investigadores en las áreas de ciencias sociales. Ahora bien, dos estudios con características similares, realizados con diez años de diferencia, llegaron a conclusiones distintas. Por un lado Folster (1989) afirma que las revistas académicas son considerablemente más importantes, y por consiguiente, de mayor uso para los investigadores de estas áreas. Por su lado, Calva González (1999) define a las monografías como la principal fuente de información de los investigadores en ciencias sociales. Ambos estudiosos utilizaron la encuesta como herramienta para obtener la opinión de los propios investigadores, aunque es necesario reflexionar sobre su uso en este tipo de estudios, ya que las consultas reales pueden diferir respecto a lo que un investigador declara en un cuestionario, cuando no tiene tiempo o interés en responder. Aún más, los investigadores suelen usar con frecuencia los recursos de información que tienen disponibles en su institución académica, pero no se debe descartar que también utilizan muchos que no necesariamente están en sus bibliotecas.

\section{País de publicación}

De acuerdo con nuestro estudio, y como se muestra en el cuadro 6, los documentos publicados en México (38.91\%) son los más citados por los autores de $E D U$, seguidos por los editados en Estados Unidos $(25.14 \%)$. Aunque en la revista habitualmente publican autores de diferentes países, es indudable que la mayoría de ellos son mexicanos, o bien, extranjeros que radican en México, por lo que es evidente en

${ }^{2}$ La literatura gris es definida como aquella que no ha sido publicada por los canales habituales de producción de la industria editorial, por lo que tampoco alcanza los medios de comercialización y distribución que ésta tiene. Estas características hacen que este tipo de publicaciones sea difícil de localizar y, por consiguiente, de adquirir. 


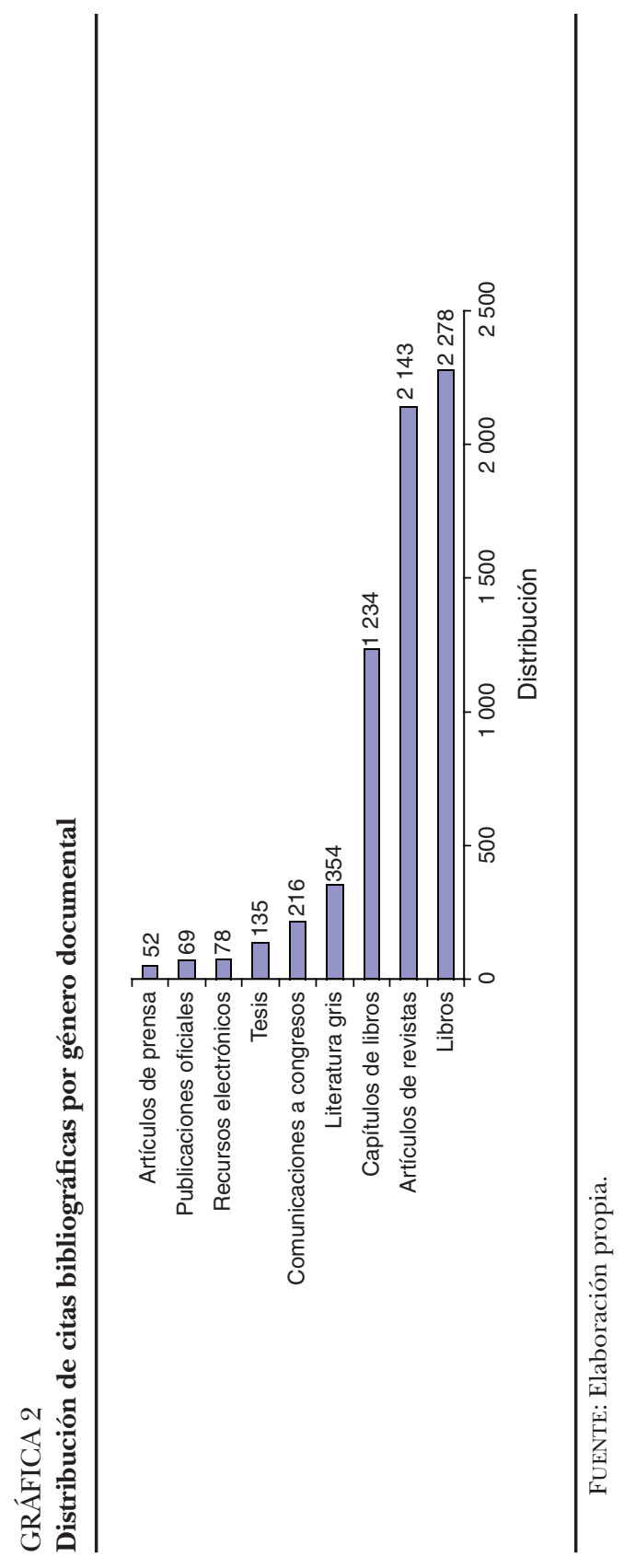




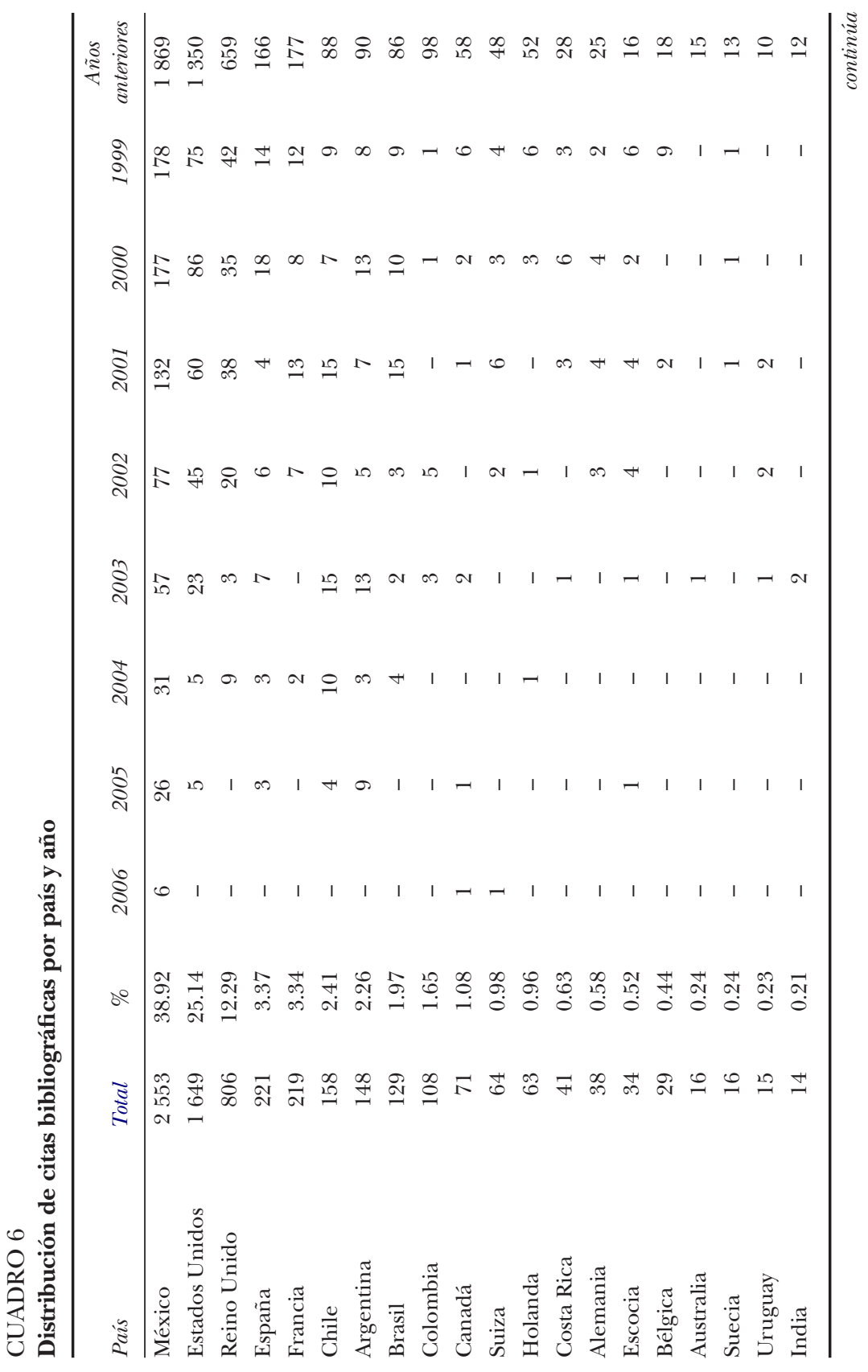




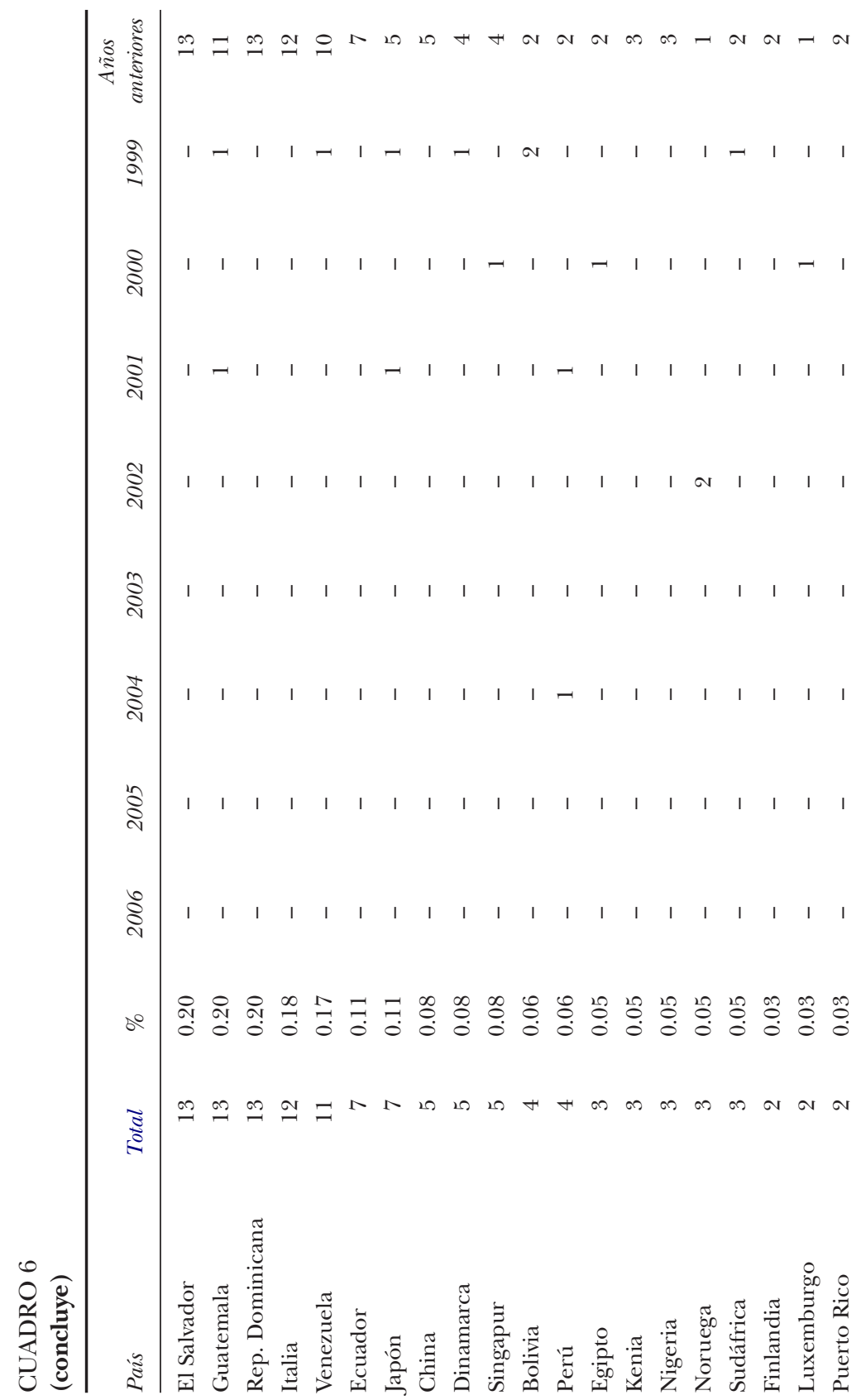




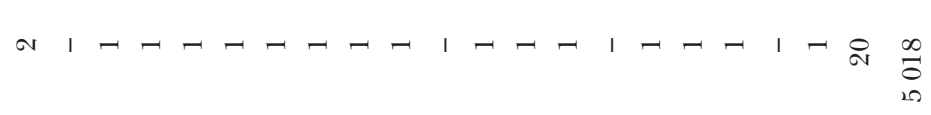

$$
\begin{aligned}
& \begin{array}{llllllllllllllllllllll}
1 & 1 & 1 & 1 & 1 & 1 & 1 & 1 & 1 & 1 & 1 & 1 & 1 & 1 & 1 & 1 & 1 & 1 & 1 & 1 & 0 & 8 \\
\hline
\end{array}
\end{aligned}
$$

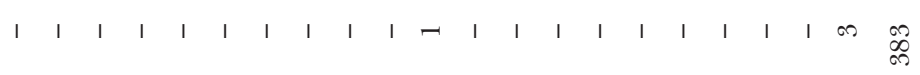

$$
\begin{aligned}
& \begin{array}{llllllllllllllllllllll}
1 & 1 & 1 & 1 & 1 & 1 & 1 & 1 & 1 & 1 & 1 & 1 & 1 & 1 & 1 & 1 & 1 & 1 & - & 1 & -\frac{a}{2}
\end{array} \\
& \begin{array}{llllllllllllllllllllll}
1 & 1 & 1 & 1 & 1 & 1 & 1 & 1 & 1 & 1 & 1 & 1 & 1 & 1 & 1 & 1 & 1 & 1 & 1 & 1 & 1 & 0 \\
2
\end{array} \\
& \begin{array}{llllllllllllllllllllll}
1 & 1 & 1 & 1 & 1 & 1 & 1 & 1 & 1 & 1 & 1 & 1 & 1 & 1 & 1 & 1 & 1 & 1 & 1 & 1 & 1 & 0 \\
2
\end{array} \\
& \begin{array}{lllllllllllllllllllllll}
1 & -1 & 1 & 1 & 1 & 1 & 1 & 1 & 1 & 1 & 1 & 1 & 1 & 1 & - & 1 & 1 & 1 & 1 & 1 & 1 & 5
\end{array} \\
& \begin{array}{llllllllllllllllllllll}
1 & 1 & 1 & 1 & 1 & 1 & 1 & 1 & 1 & 1 & 1 & 1 & 1 & 1 & 1 & 1 & 1 & 1 & 1 & 1 & 1 & 9
\end{array} \\
& \begin{array}{llllllllllllllllllllll}
1 & 1 & 1 & 1 & 1 & 1 & 1 & 1 & 1 & 1 & 1 & 1 & 1 & 1 & 1 & 1 & 1 & 1 & 1 & 1 & 1 & \infty
\end{array}
\end{aligned}
$$

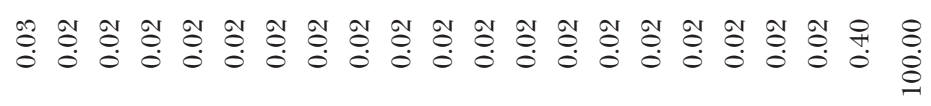

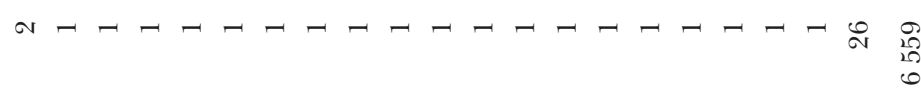

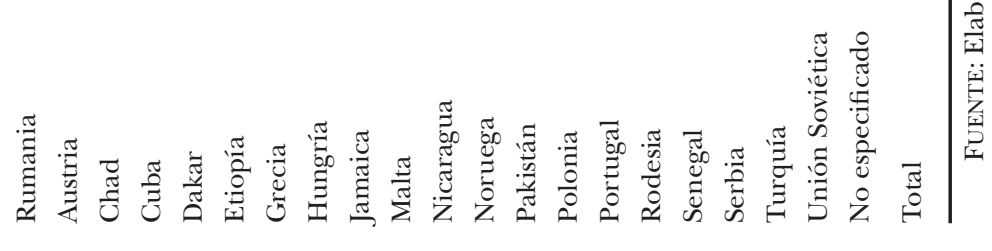


los resultados del estudio la accesibilidad que tienen a los documentos que se publican aquí, frente a los que se publican en otros países. Son significativas las citas procedentes de países como Francia, España, Chile y Argentina, en los que se publica una gran cantidad de textos sobre temáticas afines a las que aborda $E D U$, por ejemplo, las publicaciones de la Comisión Económica para América Latina y el Caribe (CEPAL), con sede en Santiago de Chile, y cuyas publicaciones son muy consultadas por los autores de $E D U$. En total el cuadro 6 refleja la producción de 61 países, aunque en algunos casos sea mínima.

El cuadro 7 nos muestra la relación que existe entre el total de citas por país y el género documental al que pertenece cada uno de los documentos citados. Como se observa, los libros publicados en México (903) fueron consultados en mayor medida que los de Estados Unidos (553). En el caso de los artículos de revista la situación es a la inversa: hay mayor consumo de los artículos publicados por las editoriales estadounidenses (679). Con excepción de Chile, Argentina y en menor medida Brasil, el resto de los países de América Latina tiene una mínima representatividad. Las razones pueden ser diversas, pero cabe mencionar la que quizá es la más importante: el mercado editorial en América Latina es muy deficiente en la comercialización y la distribución, lo que impide una adecuada circulación de los materiales entre los países de la región. En el cuadro 6 se aprecia que los investigadores tienen poco acceso a los documentos que se publican en esos países, lo que por supuesto no es equivalente a falta de interés.

\section{Revistas más citadas}

$E D U$ sobresale como la fuente más citada por los autores que publicaron en este periodo, con 149 citas (cuadro 8). Como se mencionó al inicio de este documento, $E D U$ ha destacado desde hace varias décadas como una revista de alta calidad académica, por lo que no es casualidad que en estos temas sea considerada fuente primordial de información. Cabe mencionar, sin embargo, que algunas de las revistas que aparecen en el cuadro 8 tienen un alto número de citas debido a que la mayoría de éstas fueron hechas en un solo artículo, como es el caso de Conciencia Latinoamericana, que obtuvo 18 citas de un mismo artículo. Algunos autores recurren a esta práctica cuando buscan profundizar sobre un tema en el que un solo título de revista puede aportarle información muy relevante para su investigación. 


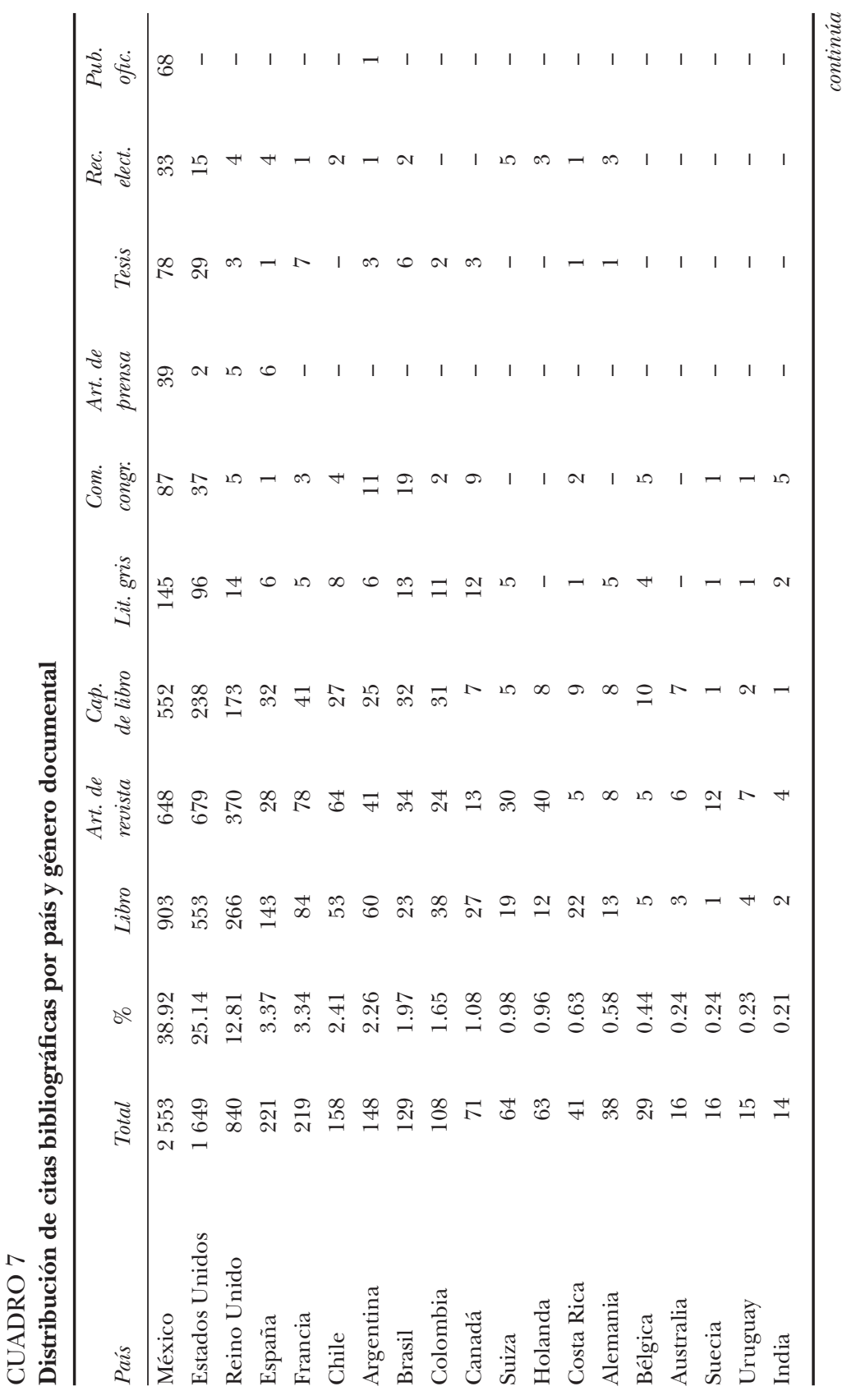




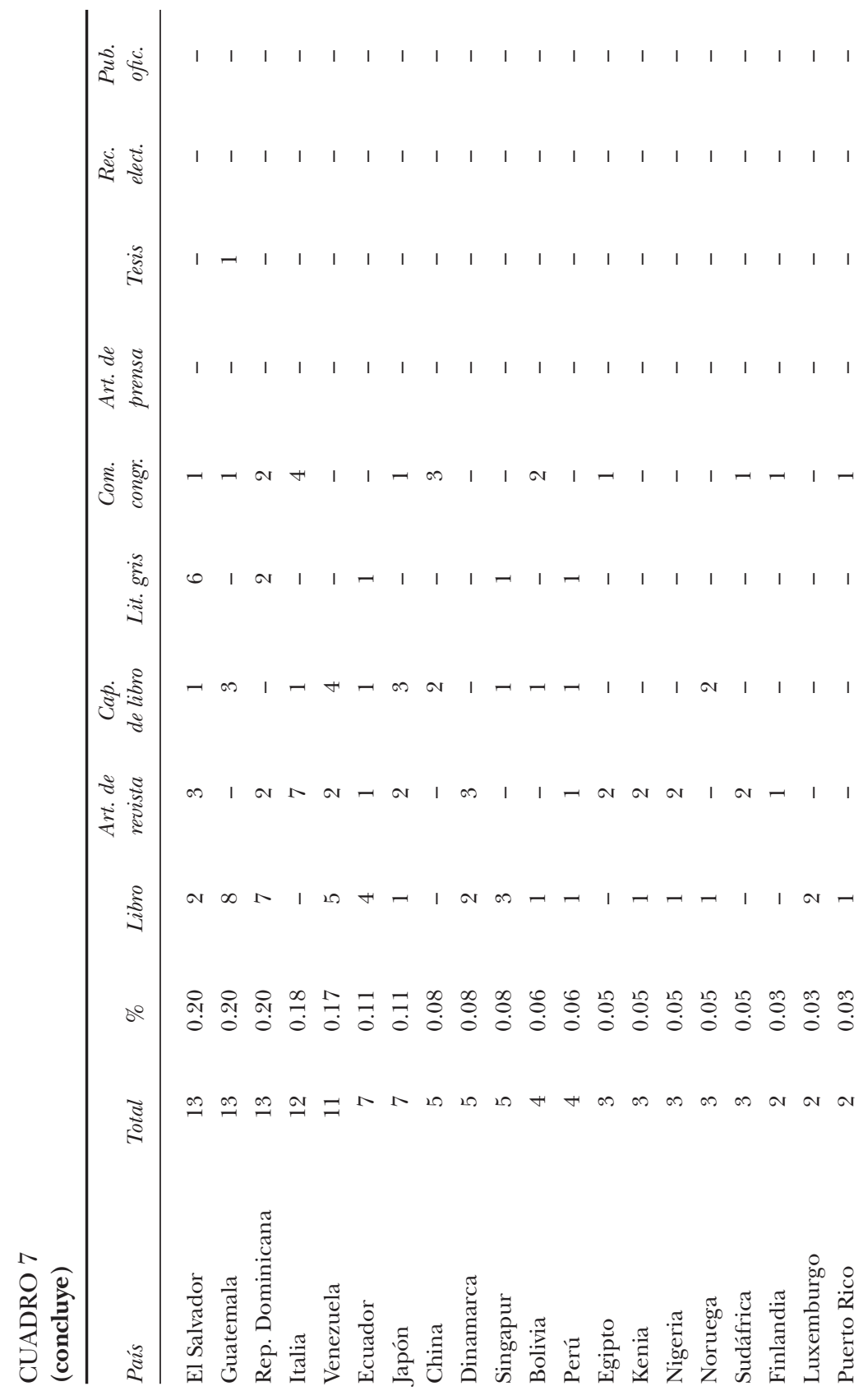




$$
\begin{aligned}
& \begin{array}{llllllllllllllllllllll}
1 & 1 & 1 & 1 & 1 & 1 & 1 & 1 & 1 & 1 & 1 & 1 & 1 & 1 & 1 & 1 & 1 & 1 & 1 & 1 & 8
\end{array} \\
& \begin{array}{llllllllllllllllllllll}
1 & 1 & 1 & 1 & 1 & 1 & 1 & 1 & 1 & 1 & 1 & 1 & 1 & 1 & 1 & 1 & 1 & 1 & 1 & 1 & 4 & 0
\end{array}
\end{aligned}
$$

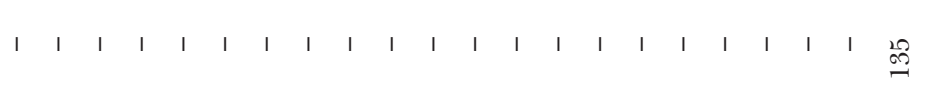

$$
\begin{aligned}
& \begin{array}{llllllllllllllllllllll}
1 & 1 & 1 & 1 & 1 & 1 & 1 & 1 & 1 & 1 & 1 & 1 & 1 & 1 & 1 & 1 & 1 & 1 & 1 & 1 & 1 & 0 \\
0
\end{array}
\end{aligned}
$$

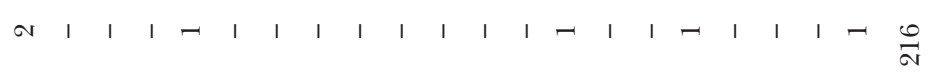

$$
\begin{aligned}
& \begin{array}{llllllllllllllllllllll}
1 & 1 & 1 & 1 & 1 & 1 & 1 & 1 & 1 & 1 & 1 & 1 & 1 & 1 & 1 & 1 & 1 & 1 & 1 & 1 & \infty & \frac{0}{20}
\end{array}
\end{aligned}
$$

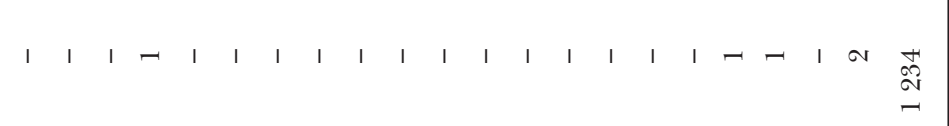

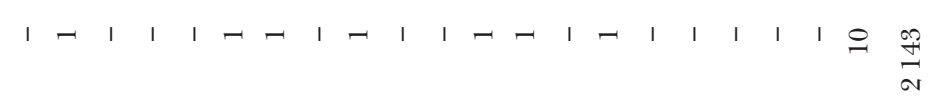

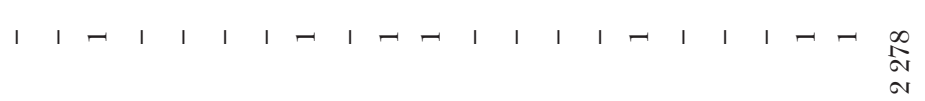

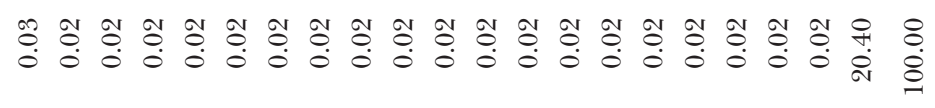

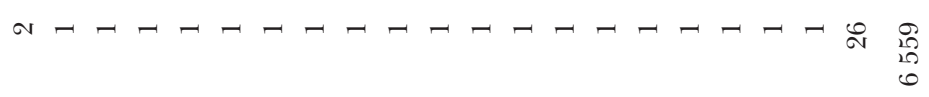

$$
\begin{aligned}
& \text { 光 }
\end{aligned}
$$


En total se citaron 637 títulos de revistas en EDU, de los cuales sólo 15 recibieron 22 o más citas; las 615 restantes obtuvieron 21 o menos citas. En el cuadro 8 se aprecia que los autores recurren sólo de forma esporádica a artículos de reciente publicación para sus investigaciones, al grado de que en todo el estudio, inclusive en los demás géneros documentales, sólo se registra una cita a una obra publicada en 2006.

\section{Libros más citados}

Con la finalidad de identificar los libros más citados por los autores de $E D U$ durante este periodo se preparó una lista con todos los títulos de las obras monográficas, incluyendo aquéllos de los que sólo fue citado un capítulo. Es decir, se hizo un conteo de títulos de libros independientemente de que hayan sido citados de forma completa o sólo por alguno de sus capítulos, aunque en el cuadro 5 aparecen por separado. Como se aprecia en el cuadro 9, de este análisis se obtuvo un total de 2579 títulos de libros, de los cuales sólo 11 fueron citados en 10 o más ocasiones, mientras que los restantes 2568 fueron citados en 9 o menos veces, lo que produjo un total de 3513 citas para este tipo de documentos. En la lista destacan las obras publicadas en español, lo que denota una gran preferencia por este idioma en ciertos temas y por parte de varios autores. Un tratamiento especial merecen las publicaciones editadas por el Instituto Nacional de Estadística, Geografía e Informática (INEGI) y el Consejo Nacional de Población (Conapo) de México, cuyos títulos reciben una gran cantidad de citas ya que son materia prima para los investigadores de estas áreas, principalmente para los demógrafos y para los urbanistas. Indudablemente su mayor fortaleza en este campo son los resultados de los censos de población y el análisis de la situación migratoria, de los que emana una serie de obras de gran demanda para los especialistas.

En el cuadro 10 se muestra la frecuencia absoluta de los títulos citados por los autores durante el periodo. En éste se aprecia que una gran mayoría de los documentos (2 131) sólo fueron citados en una ocasión; el mayor número de citas que alcanzó un título fue de 20, siendo éste el único caso que registró tal cifra. 


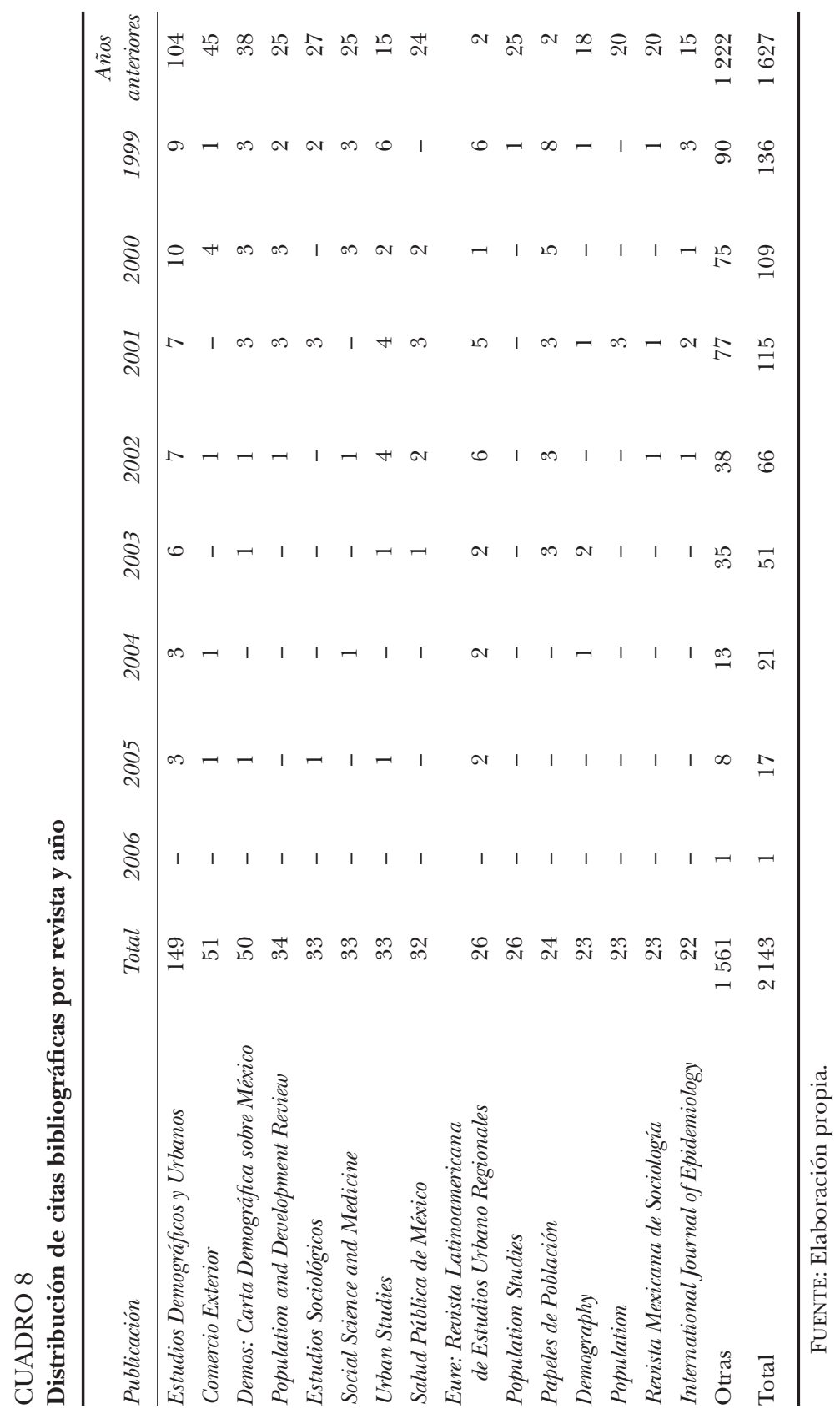




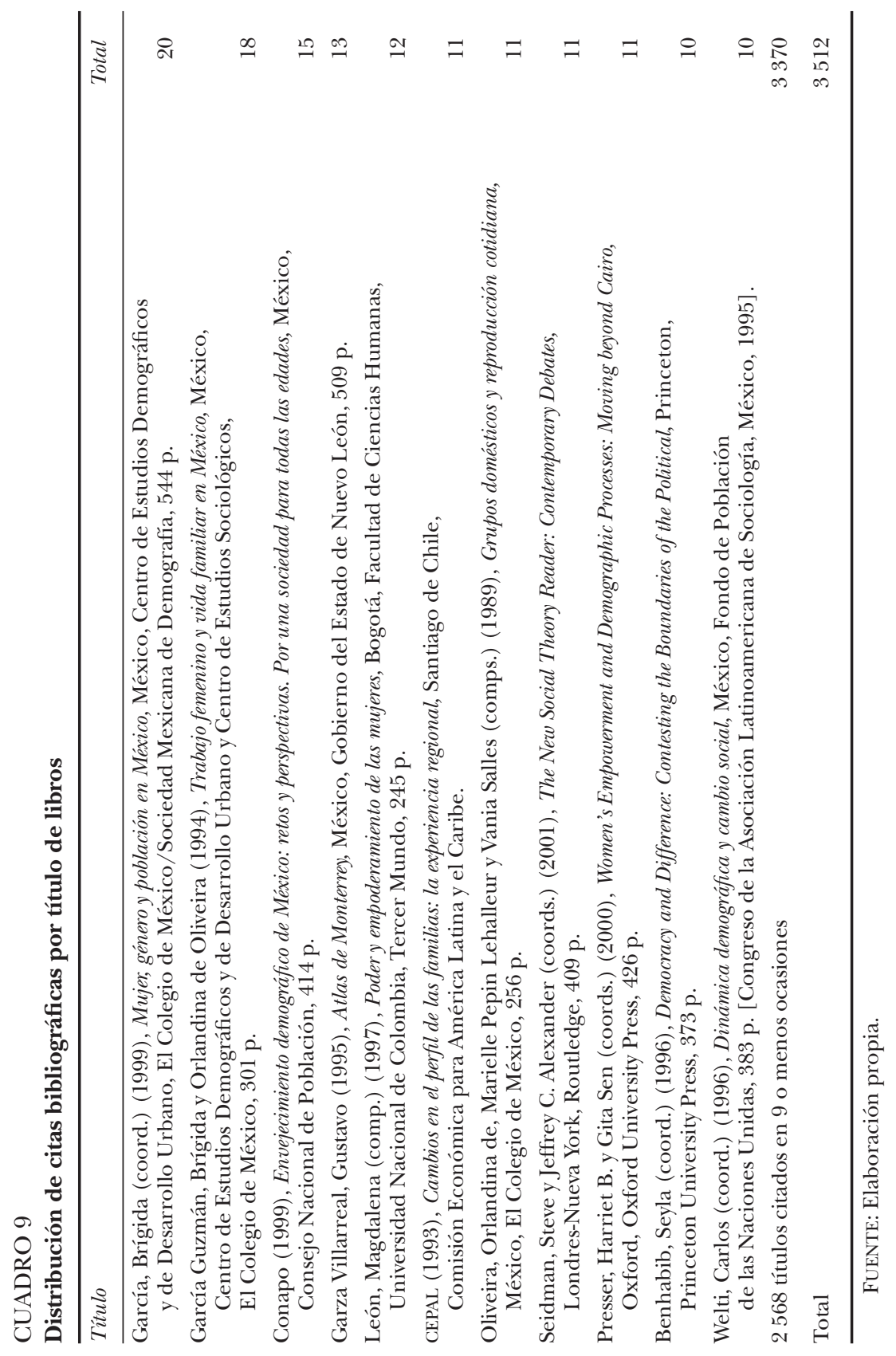


CUADRO 10

Distribución de trabajos por frecuencia de citas

\begin{tabular}{cc}
\hline Número de veces que fue citado & Frecuencia absoluta (títulos citados) \\
\hline 20 & 1 \\
18 & 1 \\
15 & 1 \\
13 & 1 \\
12 & 1 \\
11 & 4 \\
10 & 2 \\
9 & 4 \\
8 & 4 \\
7 & 8 \\
6 & 9 \\
5 & 18 \\
4 & 39 \\
3 & 106 \\
2 & 249 \\
1 & 2131 \\
General & 2579 \\
\hline
\end{tabular}

FUENTE: Elaboración propia.

\section{Idioma}

Algunos estudios, como el realizado por Romanos de Tiratel (2000), demuestran que los investigadores en las áreas de ciencias sociales y humanidades prefieren consultar principalmente fuentes en su mismo idioma. En el cuadro 11 se aprecia esta preferencia al observar que un poco más de la mitad de los documentos consultados por los autores que publican en $E D U$ se encuentra en español (51.55\%), aunque los textos en inglés también tienen un porcentaje muy alto $(43.33 \%)$. Los escritos en francés y portugués son consultados en menor medida (3.11 y $1.57 \%$, respectivamente), mientras que la consulta de documentos en otros idiomas es mínima.

Es importante considerar que algunos de los investigadores del CEDUA se han formado en universidades francófonas, por lo que inferimos que sean quienes más consulten documentos redactados en francés. Por otra parte, algunos de los autores que publicaron durante 


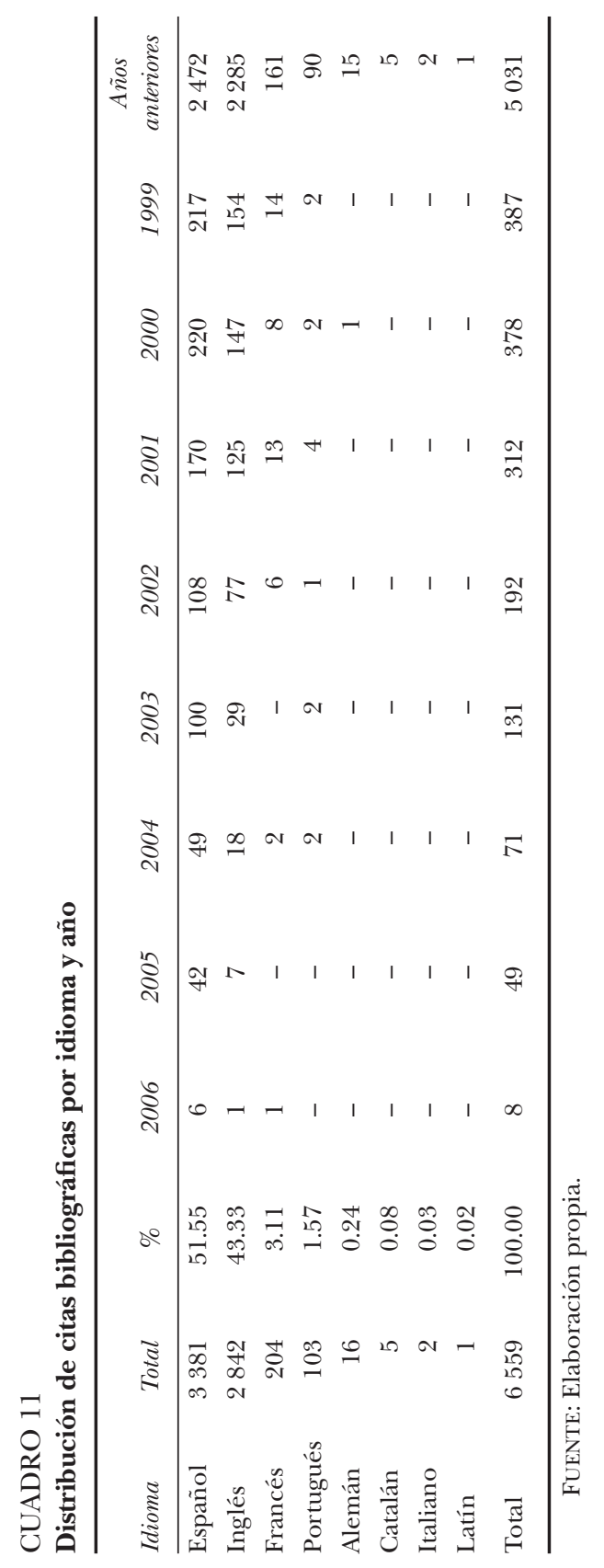




\section{GRÁFICA 3}

\section{Distribución de citas bibliográficas por idioma}

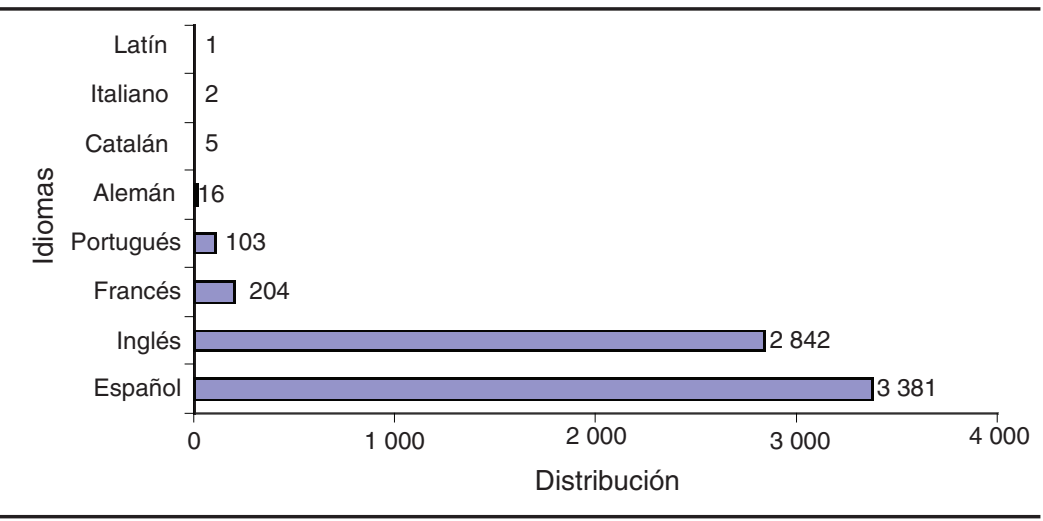

FUENTE: Elaboración propia

este periodo en $E D U$, tienen como idioma nativo el portugués, por lo que es probable que sean ellos quienes hayan citado el mayor número de documentos en dicho idioma. Desde luego que no podemos dejar a un lado a los investigadores que, sin haberse formado o sin ser nativos de países francófonos o de habla portuguesa, leen documentos en esos idiomas.

\section{Actualidad de los trabajos}

Para medir la actualidad de los documentos consultados, se recurrió a calcular el índice de Price, ${ }^{3}$ de acuerdo con la metodología empleada por Pérez Andrés, Estrada Lorenzo, Villar Álvarez y Rebollo Rodríguez (2007), es decir, a partir del porcentaje de citas con menos de cinco años de antigüedad. Como se observa en el cuadro 12, de los diez años analizados, 1999 es en el que se utilizó la literatura con más actualidad $(35.79 \%)$, en tanto que 2007 es el que tiene menos literatura actualizada $(15.84 \%)$. Se observa que a partir de 1999 se ha producido un descenso en la actualidad de los documentos consultados por los investigadores. Asimismo, en 1999, 2006 y 2007, ningún autor refirió en sus textos algu-

${ }^{3} \mathrm{El}$ índice de Price es un indicador que mide la actualidad de las citas en un conjunto de documentos o en una disciplina, haciendo énfasis en aquellos que tienen una antigüedad menor a cinco años, respecto del total de las referencias. 
na fuente publicada en el mismo año. Como muestra el cuadro 12, en los diez años que se analizaron de la revista sólo se localizaron 23 citas a documentos publicados en el mismo año en que fueron citados. De esta forma, del total de 6559 citas analizadas, se contabilizaron 1753 citas con menos de cinco años de antigüedad, 26.73\% del total de las citas.

Como se aprecia en el cuadro 13, el índice de Price varía significativamente en cada uno de los géneros documentales. La mayor actualidad se concentra en los artículos de prensa $(84.61 \%)$, en tanto que los libros, el género que mostró mayor uso por parte de los investigadores, se colocan como los menos actualizados (20.10\%).

\section{Autores más citados}

En el recuento de las citas a los autores se omitieron todas las autocitas que se localizaron en la base de datos debido a que varios autores se autocitaban $n$ cantidad de veces, evitándose así que dos o tres de ellos encabezaran la lista de los más citados. Más aún, hubo casos en que las repetidas citas a un autor eran en realidad autocitas provenientes de un solo artículo.

Después de haber eliminado las autocitas se obtuvo una lista de 4293 autores (cuadro 14). De ellos, 21 fueron citados en 15 o más ocasiones durante el periodo, entre los que destacan seis investigadores del CEDUA, y una autora del Centro de Estudios Sociológicos (CES) de El Colegio de México, quien encabeza la lista. Además de que sus trabajos despiertan el interés de otros autores, la frecuente inclusión de dicha autora puede deberse a que algunos de sus documentos han sido escritos en coautoría con investigadores del propio CEDUA.

En este rubro también se identificaron 4272 autores con 14 o menos citas en la revista, con un total de 7194 citas de autor en el periodo estudiado.

\section{Núcleos de Bradford}

Para elaborar los núcleos de Bradford ${ }^{4}$ se consideraron las 2143 referencias procedentes de 637 títulos de revistas. El núcleo principal lo

${ }^{4}$ Los núcleos de Bradford (Ley de Bradford) representan un indicador de la dispersión de la información científica; su formulación muestra que si las revistas en un área temática se dividen en terciles, entonces el número de revista en cada grupo será proporcional a 1:n:n², y donde el núcleo principal representa el conjunto de revistas de mayor pertinencia para un área del conocimiento (Sanz-Valero et al., 2010). 


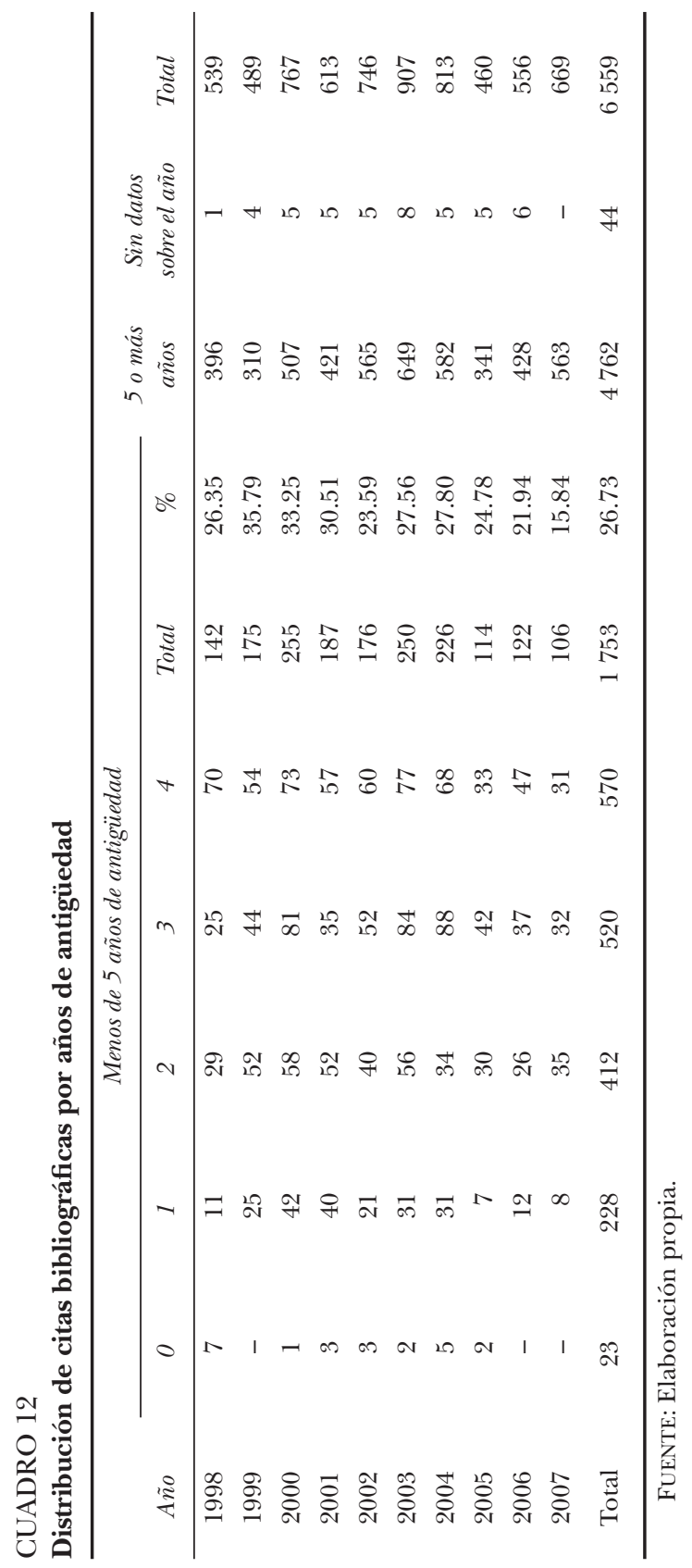




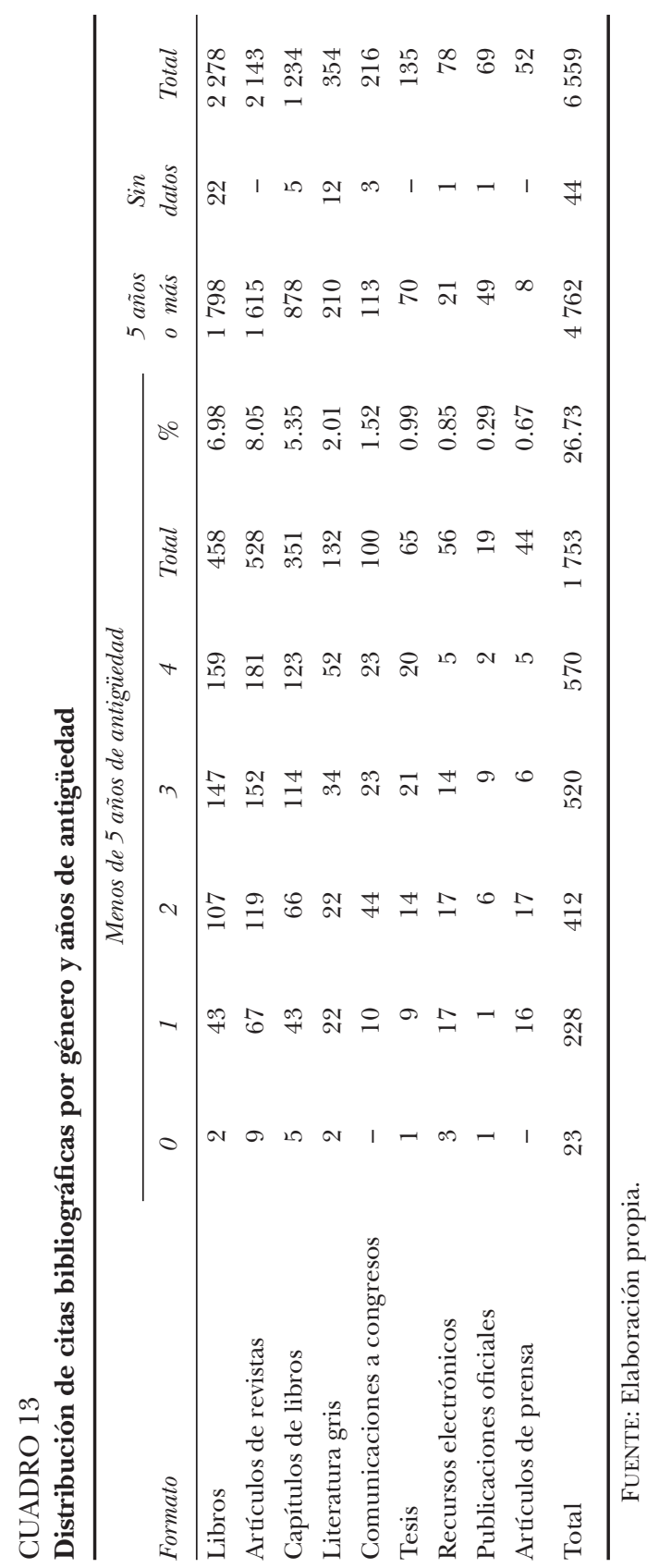


CUADRO 14

Distribución de citas bibliográficas por autor

\begin{tabular}{ccc}
\hline Autor & Centro de adscripción & Total de citas \\
\hline 1 & CES & 75 \\
2 & CEDUA & 69 \\
3 & & 27 \\
4 & CEDUA & 26 \\
5 & CES & 25 \\
6 & CEDUA & 25 \\
7 & & 22 \\
8 & & 21 \\
9 & & 21 \\
10 & CES & 21 \\
11 & & 20 \\
12 & CES & 19 \\
13 & & 18 \\
14 & & 18 \\
15 & & 17 \\
16 & CEDUA & 17 \\
17 & & 17 \\
18 & CEDUA & 16 \\
19 & CES & 16 \\
20 & & 15 \\
21 & CEDUA & 15 \\
4272 autores con 14 o menos citas & & 6674 \\
autores & & 7194 \\
\hline
\end{tabular}

FUENTE: Elaboración propia.

forman 22 revistas, entre las que se encuentran ocho mexicanas. Las 22 revistas suponen $3.43 \%$ de las revistas fuente y agrupan 718 referencias. Por su parte, con un número similar de referencias (716 y 719 respectivamente), el núcleo 2 está conformado por 100 títulos de revistas, en tanto que el núcleo 3 por 515 . El cuadro 15 representa la distribución de las referencias en estas tres zonas.

\section{Futuros estudios}

El estudio realizado nos demuestra que con base en esta metodología se pueden obtener resultados objetivos que nos permitan tomar decisiones en el área de desarrollo de colecciones de la Biblioteca con la 


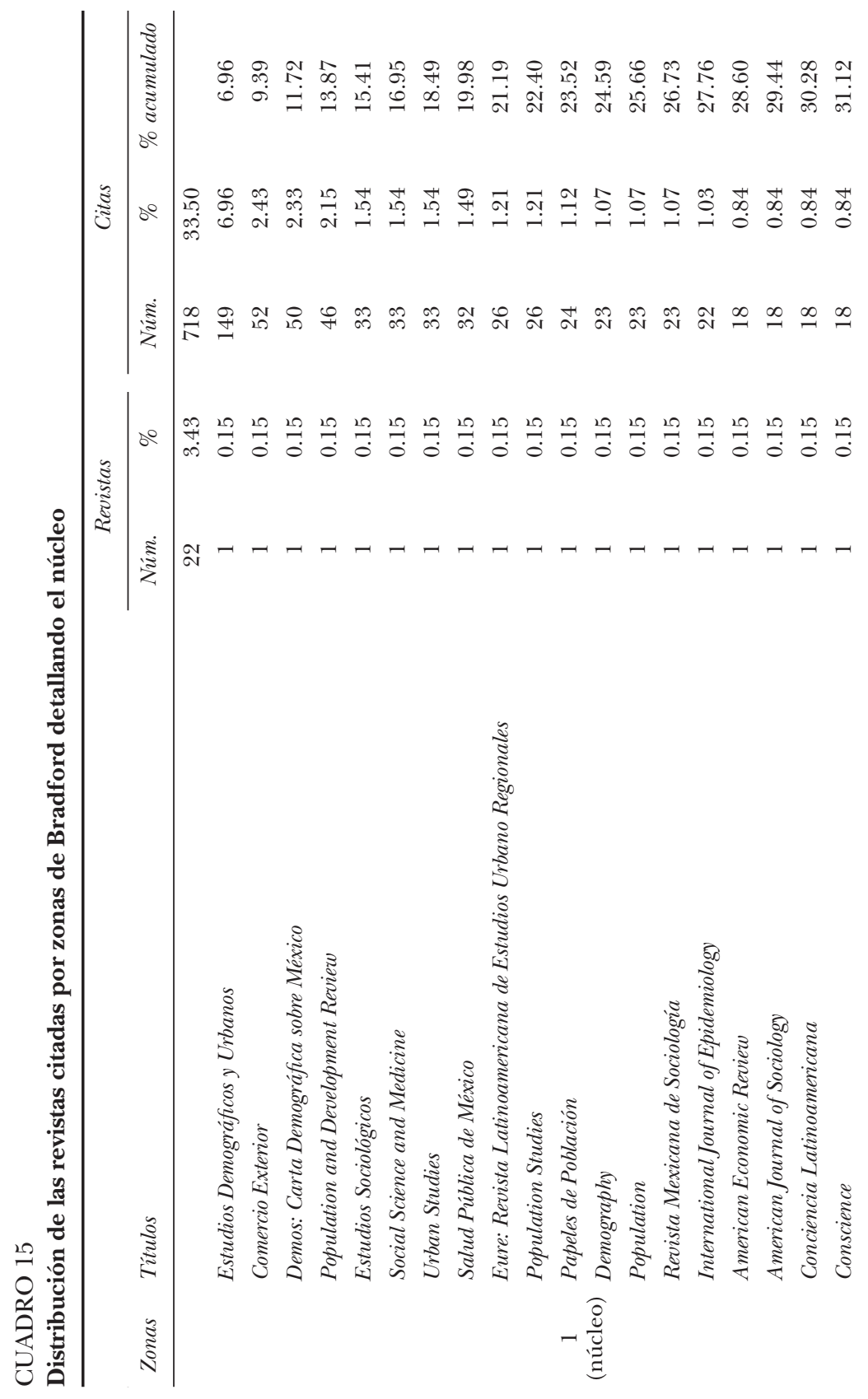




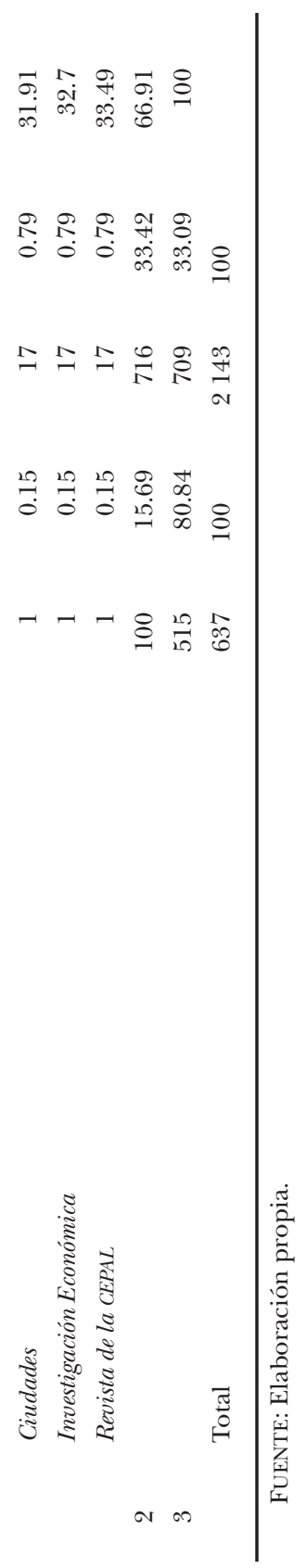


finalidad de optimizar los recursos. Un estudio posterior nos brindaría, por ejemplo, la posibilidad de obtener patrones en el consumo de información de colecciones específicas, tal es el caso de las publicaciones periódicas o de los libros. Como lo demuestran los estudios que ya se han llevado a cabo en muchas bibliotecas, aplicar esta metodología a grupos de investigadores de un área determinada suele ser de gran utilidad para conocer con más precisión sus hábitos en el uso de la información. En nuestro caso, podríamos elegir entre los demógrafos, los urbanistas o los especialistas en medio ambiente.

\section{Conclusiones}

Un estudio de esta naturaleza no es suficiente para reorientar por completo las políticas de desarrollo de colecciones de una biblioteca debido a que sólo hemos analizado uno de los muchos títulos de las publicaciones que existen en esta área. A pesar de lo anterior, los resultados pueden ser útiles para confirmar o reconsiderar algunas de las políticas establecidas por la Biblioteca.

La Biblioteca Daniel Cosío Villegas invierte cada año cientos de miles de pesos de su presupuesto de adquisiciones en suscripciones a revistas electrónicas y bases de datos cuya consulta no se refleja en este estudio. De acuerdo con los datos obtenidos, es muy pobre el uso que los investigadores están haciendo de los recursos electrónicos. Sin embargo, ellos frecuentemente demandan dichos recursos de manera personal. El problema es que no lo reflejan en sus textos; la mayoría cita sus fuentes como si se tratara de documentos impresos, a pesar de que en muchas ocasiones las obtuvieron de un medio electrónico. Por tanto, se deberá diseñar otra herramienta para medir el efecto de las nuevas tecnologías en su investigación.

Aunque un estudio más amplio podría confirmar con mayor exactitud los datos que aquí hemos obtenido, es evidente que la mayoría de los investigadores de estas áreas prefiere consultar los documentos publicados en español e inglés, y con menor frecuencia, aunque no por ello menos relevante, recurren a publicaciones editadas en francés y portugués. Por tal motivo la política de desarrollo de colecciones debe orientarse hacia esos idiomas, evitando en la medida de lo posible la incorporación de títulos en otras lenguas, cuyo contenido muy probablemente no será suficientemente aprovechado.

La accesibilidad a los documentos tiene especial relevancia para 
los investigadores. Se observó que en gran medida la poca o nula consulta a materiales de países como los latinoamericanos se debe a deficiencias en los procesos de comercialización y distribución de las publicaciones. La Biblioteca Daniel Cosío Villegas debe reforzar los convenios de canje y donación que mantiene con bibliotecas de países que tienen poca representatividad en este tipo de estudios, ya que muchas veces es sólo mediante dichos convenios que se puede acceder a sus publicaciones.

Por la naturaleza de su trabajo académico, los investigadores, en especial los demógrafos, hacen un uso recurrente de fuentes estadísticas y censales editadas por los organismos encargados de realizar dichos estudios en los diferentes países. En el caso de México, destaca la consulta a las publicaciones del Instituto Nacional de Estadística, Geografía e Informática (INEGI) y del Consejo Nacional de Población (Conapo). La Biblioteca Daniel Cosío Villegas es una unidad depositaria de las publicaciones del INEGI, por lo que es necesario mantener el cuidado necesario para que éstas ingresen sin contratiempos al acervo, se procure su actualización permanente y se hagan las reclamaciones necesarias cuando los materiales no hayan sido suministrados por el propio Instituto. Se sugiere llevar a cabo las mismas acciones con las publicaciones del Conapo.

\section{ANEXO}

\section{Lista de las revistas citadas en $E D U$ en el periodo $1998-2007$}

Access

Acta Cardiologica

Acta Obstetrica et Gynecologica Scandinavica

Acta Paediatrica Supplementum

Acta Sociológica

Acta Tropica

Administration and Society

Administrative Science Quarterly

Advanced Applied Probabilities

African Journal of Reproductive Health

Ageing and Society

Aggression and Violent Behavior

AIDS 
AIDS and Behavior

AIDS Care

Alpanchis

Alteridades

Alternativas para el Desarrollo

Ambiente y Desarrollo

Ambiente: Ética y Estética para el Ambiente Construido

América Indígena

American Anthropologist

American Behavioral Scientist

American Economic Review

American Journal of Agricultural Economics

American Journal of Economics and Sociology

American Journal of Nursing

American Journal of Obstetrics and Gynaecology

American Journal of Occupational Theraphy

American Journal of Physical Anthropology

American Journal of Political Science

American Journal of Public Health

American Journal of Sociology

American Journal of Tropical Medicine and Hygiene

American Political Science Review

American Psychologist

American Sociological Review

American University Law Review

Annales de la Societé Belge de Médecine Tropicale

Análisis Económico

Annales Chirurgiae et Gynaecologiae Supplementum

Annales de Demographie Historique

Annales Pharmaceutiques Françaises

Annals of Epidemiology

Annals of Mathematical Statistics

Annals of Operations Research

Annals of Regional Science

Annals of Statistics

Annals of the American Academy of Political and Social Science

Annals of the Association of American Geographers

Annals of the New York Academy of Sciences

Annals of Tropical Medicine and Parasitology

Annual Review of Anthropology 
Annual Review of Ecological Systems

Annual Review of Energy and Environment

Annual Review of Sociology

Anthropological Quarterly

Anuario de Estudios Centroamericanos

Anuario de Estudios Urbanos

Applied Economics

Archives of Gynaecology and Obstetrics

Archivos Latinoamericanos de Nutrición

Argumentos

Atención Primaria

Bell Journal of Economics

Biometrics

Biometrika

BJOG: an International Journal of Obstetrics and Gynaecology

Boletín de Estadistica

Boletín de Información Estadistica, Recursos y Servicios

Boletín de la Asociación Demográfica Histórica

Boletín de la Oficina Sanitaria Panamericana

Boletín de la Sociedad Matemática Mexicana

Boletín del Instituto de Geografía

Boletín Demográfico

Boletin Editorial de El Colegio de México

Boletín Informático

Boletín Médico del Hospital Infantil de México

Bosques, Árboles y Comunidades Rurales

Brazilian Journal of Population Studies

British Journal of Mathematical and Statistical Psychology

British Journal of Obstetrics and Gynaecology

British Journal of Sociology

British Medical Bulletin

British Medical Journal

Bulletin of Latin America Research

Bulletin of the World Health Organization

Cadernos da FEA

Cahiers de Géographie du Québec

Cahiers de l'ORSTOM

Cahiers des Amériques Latines

Cahiers des Sciences

Cahiers d'Études Africaines 
Cahiers Québécois de Démographie

Cahiers Sciences Humaines

Cairo Paper in Social Sciences

Calidad Ambiental

Cambridge Journal of Economics

Canadian Geographer

Canadian Journal of Public Health

Canadian Public Administration

Canadian Review of Sociology and Anthropology

Canadian Studies in Population

Carta sobre Población

Central African Journal of Medicine

Challenge

Ciencia

Cities

Ciudad y Territorio: Estudios Territoriales

Ciudades

Comercio Exterior

Comparative Education Review

Comparative Political Studies

Comunic, Saúde, Educ

Conciencia Latinoamericana

Con-Ciencia Política

Congressional Quarterly Weekly Report

Conscience

Contraception

Contribuciones

Convergencia

Corett Informa

Coyuntura: Análisis y Debate de la Revolución Democrática

Cuadernos Agrarios

Cuadernos Americanos

Cuadernos de Antropología

Cuadernos de Economía

Cuadernos de Saúde Pública

Cuadernos del Consumidor

Cuadernos Médicos Sociales

Culture and Society

Culture, Health and Sexuality

Culture, Medicine and Psychiatry 
Current Anthropology

Current Opinion in Pulmonar Medicine

Dados

Daedalus

Debate Feminista

Debates: Revista de la Ciudad de México

Demografía y Economía

Demographic Research

Demographie

Demography

Demos: Carta Demográfica sobre México

Desarrollo Económico

Desarrollo y Sociedad

Desastres y Sociedad

Development and Change

Development and Culture Change

Development in Practice

Diabetic Medicine: a Journal of the British Diabetic Association

Disasters

Discover

Divulgação em Saúde para Debate

Documentación Administrativa

East African Medical Journal

Eastern Mediterranean Health Journal

East-West Center Working Paper

Econometrica

Economía Abierta

Economia Brasileira em Perspectiva

Economia Colombiana

Economía Informa

Economía Mexicana

Economia, Sociedad y Territorio

Economia: Journal of the Latin American and Caribbean Economic Association

Economía: Teoría y Práctica

Economic Development and Cultural Change

Economic Development Review

Economic Geography

Economic Journal

Economic Letters

Economic Record 
Economica

Economics on Education Review

Economie Rurale

Educación, Productivdad y Empleo

Ekistics

El Cotidiano

El Jarocho Verde

El Mercado de Valores

El Salvador en Construcción

El Trimestre Económico

Electoral Studies

Emerging Infectious Diseases

Employment and Society

Environment

Environment and Behavior

Environment and Planning

Environment and Planning $A$

Environment and Planning $B$

Environment and Planning $D$

Environment and Urbanization

Epidemiology

Espace, Populations, Sociétés

Espaco e Tempo: Revista de Geografia da USP

Espiral: Estudios sobre Estado y Sociedad

Esprit

Estadistica

Estadistica y Economía

Este País

Estudios

Estudios Agrarios

Estudios de Trabajo

Estudios Demográficos y Urbanos

Estudios Económicos

Estudios Jaliscienses

Estudios Latinoamericanos

Estudios Migratorios

Estudios Migratorios Latinoamericanos

Estudios Sociales

Estudios Sociales Centroamericanos

Estudios Sociológicos 
Estudios Territoriales

Estudos Económicos

Etcétera

Ethiopian Medical Journal

Ethnic Racial Studies

Ethnology

Etudes Foncières

Eure: Revista Latinoamericana de Estudios Urbano Regionales

European Journal of Obstetrics, Gynecology and Reproductive Biology

European Journal of Population

European Journal of Public Health

European Review of Latin American and Caribbean Studies

European Sociological Review

Expansión

Family Process

Federalismo y Desarrollo

Feminist Economics

Feminist Studies

Foreign Affairs

Foro Internacional

Fresenius Environmental Bulletin

Frontera Norte

Futures

Gaceta

Gaceta de Economía

Gaceta Ecológica de México

Gaceta Médica de México

Gaceta UNAM

Gender and Society

Género y Sociedad

Genus

Geoforum

Geografiska Annaler

Geographica Polonica

Geographical Analysis

Geographical Journal

Geographical Review

GeoJournal

Gerontologist

Gestión Pública 
Gestión y Estrategia

Gestión y Política Pública

Ginecología y Obstetricia de México

GIRE: Boletín Trimestral sobre Reproducción Elegida

Globalización e Integración Económica Mundial

Habitat Debate

Habitat International

Health and Human Rights

Health Care Women International

Health Place

Health Policy

Health Policy and Planning

Health Reports

Health Services Management Research

Health Transition Review

Historia Mexicana

Historia, Antropología y Fuentes Orales

Historical Methods

Housing Policy Debate

Human Biology

Identities

Indian Journal of Pediatrics

Información Comercial Española

Informe Trimestral de Coyuntura

Ingeniería Hidráulica en México

International Family Planning Perspectives

International Interactions

International Journal of Anthropology

International Journal of Comparative Sociology

International Journal of Epidemiology

International Journal of Forecasting

International Journal of Geographical Information Systems

International Journal of Gynaecology and Obstetrics

International Journal of Health Services

International Journal of Tuberculosis and Lung Diseases

International Journal of Urban and Regional Research

International Labour Review

International Migration Review

International Planning Studies

International Regional Science Review 
International Review of Victimology

International Social Science Journal

International Sociology

Investigación Económica

Italian Economic Paper

Iztapalapa: Revista de Ciencias Sociales y Humanidades

JAMA

Jornal da Redesaúde

Jounal de Gynecologie, Obstetrique et Biologie de la Reproduction

Journal of American History

Journal of American Planning Association

Journal of Applied Econometrics

Journal of Biological Science

Journal of Biosocial Science

Journal of Church and State

Journal of Community Health

Journal of Comparative Family Studies

Journal of Democracy

Journal of Developing Areas

Journal of Development Economics

Journal of Development Studies

Journal of Econometrics

Journal of Economic Dynamic and Control

Journal of Economic Literature

Journal of Economic Perspectives

Journal of Economic Psychology

Journal of Economic Theory

Journal of Environmental Economics and Management

Journal of Environmental Management

Journal of Environmental Psychology

Journal of Epidemiological Community Health

Journal of Family

Journal of Family and Marriage

Journal of Family History

Journal of Family Issues

Journal of Gerontology

Journal of Gynaecology and Obstetrics

Journal of Health and Social Behavior

Journal of Health Human Services Administration

Journal of Human Evolution 
Journal of Human Hypertension

Journal of Human Resources

Journal of Hypertension

Journal of International Communication

Journal of Interdisciplinary History

Journal of Labor Economics

Journal of Marriage and Family

Journal of Mathematical Systems, Estimation and Control

Journal of Monetary Economics

Journal of Nutrition

Journal of Obstetrics and Gynaecology

Journal of Political Economy

Journal of Politics

Journal of Population Problems

Journal of Public Health

Journal of Quality in Clinical Practice

Journal of Regional Science

Journal of Sex Research

Journal of Social History

Journal of the American Institute of Planners

Journal of the American Medical Association

Journal of the American Planning Association

Journal of the American Statistical Association

Journal of the Institute of Actuaries

Journal of the Irrigation and Drainage Engineering

Journal of the Pakistan Medical Association

Journal of the Royal Statistical Society

Journal of Transport Economics and Policy

Journal of Transportation and Statistics

Journal of Transportation Engineering

Journal of Tropical Medicine and Hygiene

Journal of Tropical Pediatrics

Journal of Urban Affairs

Journal of Urban Economics

Journal of Urban Health

La Era Urbana

La Inmigración en España

La Revue du Practicien

La Ventana

Labour Force Annual Averages 
Land Economics

Landscape and Planning

Langage et Société

L'Architecture Aujourd'hui

Latin American Perspectives

Latin American Research Review

Le Monde Diplomatique

Les Annales de la Recherche Urbaine

Les Cahiers de l'Analyse

L'Espace Geographique

L'Homme

L'Ordinaire Latinoaméricain

Los Angeles Times

Marriage and Family Review

Mathematical Biosciences

Mathematical Population Studies

Medio Ambiente y Urbanización

Memoria

Mexican Studies/Estudios Mexicanos

Migración y Desarrollo

Migraciones Internacionales

Milbank Memorial Fund Quarterly

Monthly Labor Review

Monthly Review

Morbidity and Mortality Weekly Report

Mujer y Trabajo

Mujeres y Política

Mundo Urbano

Nagoya Mathematical Journal

National Geographic Research

National Journal

New England Journal of Medicine

New Internationalist

New Left Review

New Scientist

New Stateman

Nexos

Notas de Población

Notas Censales

Notas sobre la Economía y el Desarrollo 
Noticias de la Unión Europea

Novos Estudos

Nueva Antropología

Nueva Sociedad

Obstetrics and Gynecology

Oikos

Ojarasca

Oxford Economic Papers

Oxford Review of Economics Policy

Paediatric and Perinatal Epidemiology

Página

Papeles de Población

Papers in Regional Science

Papers of Proceedings of the Regional Science Association

Papers on Social Representation

Parasitology

Pasos

Pensamiento Iberoamericano

People and the Planet

Perfiles Latinoamericanos

Perspectiva Geográfica

Perspectivas Internacionales en Planificación Familiar

Perspectives on Sexual and Reproductive Health

Philosophical Transaction of the Royal Society of London

Planeación y Desarrollo

Población y Desarrollo

Política y Gobierno

Political Geography

Political Geography Quarterly

Political Power and Social Theory

Politics and Society

Population

Population and Development Review

Population and Environment

Population Bulletin

Population Bulletin of the United Nations

Population et Sociétés

Population Index

Population Reports

Population Research and Policy Review 
Population Studies

Population Studies in Archeology: a Symposium American Antiquity

Population Trends and Public Policy

Praxis International

Previdencia em Dados

Problemas del Desarrollo

Problemes d'Amèrique Latine

Proceso

Professional Geographer

Progress in Geography

Progress in Planning

Propuesta

Psychological Bulletin

Psychology and Health

Psychology Review

Public Administration Review

Public Finance Quarterly

Public Health

Quality Assurance in Health Care

Quarterly Journal of Economics

Race, Gender and Class

Recent Research Results

Recursos y Servicios: Boletín de Información Estadística

Reflexiones

Región y Sociedad

Regional and Urban Economics

Regional Science and Urban Economics

Regional Studies

Renglones

Repon on the Americas

Reproductive Health Matters

Research in Community Sociology

Review of Development Economics

Review of Economic and Statistics

Review of Economic Studies

Review of Economics and Statistics

Review of Income and Wealth

Review of International Economics

Revista A

Revista Argentina de Antropología Biológica 
Revista Brasileira de Estudos de População

Revista Ciencias

Revista Conamed

Revista de Administración Pública

Revista de Ciencias Sociales

Revista de Ciencias Sociales y Humanidades

Revista de Derecho Constitucional Español

Revista de Derecho Urbanístico y Medio Ambiente

Revista de Economía Crítica

Revista de Historia

Revista de Información y Análisis

Revista de la CEPAL

Revista de la Ciudad de México

Revista de Saúde Publica

Revista do Departamento de Geografía

Revista do Patrimonio Histórico e Artístico Nacional

Revista Encuentro

Revista Española de Investigaciones Sociológicas

Revista Española de Salud Pública

Revista Foro

Revista Geográfica

Revista Iberoamericana de Comunicación

Revista Interamericana de Planificación

Revista Interamericana de Sociología

Revista Internacional del Trabajo

Revista Latinoamericana de Ciencias Sociales

Revista Latinoamericana de Estudios del Trabajo

Revista Latinoamericana de Estudios Educativos

Revista Médica de Chile

Revista Mexicana de Ciencias Politicas y Sociales

Revista Mexicana de Sociología

Revista Realidad Económica

Revista Temas de Población

Revue d'Epidemiology et de Santé Publique

Revue Française de Science Politique

Revue Française des Affaires Sociales

Revue Internationale de Sciences Sociales

Revue Internationalle de Science Administrative

Revue Tiers Monde

Rural Sociology 
Saber Ver

Salud Pública de México

Santé

São Paulo em Perspectiva

São Paulo no Limiar do Século XXI

Scandinavian Journal of Economics

Scandinavian Journal of Social Medicine

Scandinavian Journal of Statistics

Science

Sciences Sociales et Santé

Scientific American

Scottish Journal of Political Economy

Scripta Nova: Revista Electrónica de Geografía y Ciencias Sociales

Semarnap Quincenal

Shapira Reviews

Signs

Sistema

Skandinavisk Aktuarietidskrift

Social Biology

Social Forces

Social Indicators Research

Social Problems

Social Science and Medicine

Social Science History

Social Science Journal

Social Science Quarterly

Social Science Research

Social Security Bulletin

Socialist Register

Society

Sociología del Trabajo

Sociologia Ruralis

Sociológica

Sociological Methodology

Sociological Methods and Research

Sociological Review

Sociology

Sociology of Health and Illness

South African Medical Journal

Soziale Welt 
Statistical Bulletin, Metropolitan Life Insurance Company

Stochastic Processes Applications

Studies in Family Planning

Supplement of the World Bank Economic Review

Techniques d'Enquête

Territorios: Revista de Estudios Urbanos y Regionales

Textos NEPO

The Actuary

The Economics

The Economist

The Lancet

The New York Times

Theoretical Population Biology

Third World Planning Review

Third World Quarterly

Tijdscjrift voor Economische en Sociale Geografie

Time

Topodrilo

Town Planning Review

Trabajo

Transactions

Transactions of the Actuarial Society of America

Transactions of the Institute of British Geographers

Transactions of the Manchester Statistical Society

Transport Reviews

Transportation

Transportation Research

Transportation Research A

Transportation Research Circular

Transportation Research Record

Trayectorias

Trimestre Económico

Tropical Doctor

Tropical Medicine and International Health

Urban Affairs Review

Urban Ecology

Urban Geography

Urban Studies

USA Today

Vetas 
Violence against Women

Vivienda

Voices of Mexico

Water Resources Research

West African Journal of Medicine

West Indian Medical Journal

Wold Health Forum

Women and Work

Work and Occupations

Work, Employment and Society

World Health Forum

World Health Statistics Quarterly

World Politics

\section{Bibliografía}

Aleixandre, R., J.V. Giménez Sánchez, M.L. Terrada y J.M. López Piñero (1994), "Análisis del consumo de información en la revista Medicina Clínica", Medicina Clínica, vol. 103, núm. 7, pp. 246-251.

Altmann, K.G. y G.E. Gorman (1999), "Can Impact Factors Substitute for the Results of Local Use Studies?: Findings from an Australian Case Study", Collection Building, vol. 18, núm. 2, pp. 90-94.

Ashman, A.B. (2009), "An Examination of the Research Objectives of Recent Citation Analysis Studies", Collection Management, vol. 34, núm. 2, pp. 112-128.

Aziagba, P.C. (1993), "Scientific Journal Selection Based on the Study of a Local Journal", Information Processing and Management, vol. 29, núm. 1, pp. 83-93.

Brown, K.C. (2002), "How Many Copies are Enough?: Using Citation Studies to Limit Journal Holdings”, Law Library Journal, vol. 94, núm. 2, pp. 301-314.

Burdick, A.J. y M.G. Sullivan (1993), "Citation Patterns in the Health Sciences: Implications for Serials/Monographic Fund Allocation”, Bulletin of the Medical Library Association, vol. 81, núm. 1, pp. 44-47.

Calva González,J.J. (1999), "El comportamiento en la búsqueda de información de los investigadores del área de humanidades y ciencias sociales", Investigación Bibliotecológica: Archivonomía, Bibliotecología e Información, vol. 13, núm. 27, pp. 11-40.

Díaz Grau, A., O. Mínguez y M.J. Osca Lluch (1999), "Análisis del consumo de información en los artículos españoles de terminología médica”, Boletín de la Asociación Andaluza de Bibliotecarios, vol. 14, núm. 55, pp. 19-40.

Folster, M.B. (1989), "A Study of the Use of Information by Social Science Researchers", Journal of Academic Librarianship, vol. 15, núm. 1, p. 7. 
Klassen, T.W. (2001), "Measuring Serials Usage Using Faculty Cited Journals Data”, Bottom Line, vol. 14, núm. 1, pp. 37-43.

Lascar, C. y L.D. Mendelsohn (2001), "An Analysis of Journal Use by Structural Biologists with Applications for Journal Collection”, College and Research Libraries, vol. 62, núm. 5, p. 422.

McCain, K.W. (1997), "Bibliometrics Tools for Serials Collection Management in Academic Libraries”, en C. Hepfer, T. Malinowsky y J. Gammon (coords.), Advances in Serial Management, Greenwich, JAI Press, pp. 105-146.

Meho, L.I. y S.W. Haas (2001), "Information-Seeking Behavior and Use of Social Science Faculty Stuying Stateless Nations: A Case Study”, Library and Information Science Research, vol. 23, pp. 5-25.

Nisonger, T.E. (1998), "The Application of Citation Analysis to Serial Collection Management", Management of Serials in Libraries, Englewood, Libraries Unlimited, pp. 121-156.

Ovens, C.S.H. (1995), "Citation Patterns of University of the Orange Free State Scientists”, South African Journal of Library and Information Science, vol. 63, núm. 2, p. 47.

Pérez Andrés, C., J.M. Estrada Lorenzo, F. Villar Álvarez y M.J. Rebollo Rodríguez (2007), "Estudio bibliométrico de los artículos originales de la $R \boldsymbol{e}$ vista Española de Salud Pública (1991-2000). Parte tercera: Análisis de las referencias bibliográficas", Revista Española de Salud Pública, vol. 81, núm. 3, pp. 247-259.

Romanos de Tiratel, S. (2000), "Accessing Information Use by Humanists and Social Scientists: a Study at the Universidad de Buenos Aires, Argentina”, Journal of Academic Librarianship, vol. 26, núm. 5, pp. 346-354.

Sanz-Valero, J., M.S. Ferreira, L.D. Castiel, C. Wanden-Berghe y M.C.R. Guilam (2010), El filtro académico brasileño: aplicación a la literatura científica sobre actividad física, Revista de Saúde Pública, julio, doi:10.1590/S003489102010005000024 (publicación electrónica previa a la edición impresa).

Soehner, C.B. y D.T. Richards (1992), "The Landmark Citation Method: Analysis of a Citation Pattern as a Collection Assessment Method", Bulletin of the Medical Library Association, vol. 80, núm. 4, pp. 361-366.

Sylvia, M.J. (1998), "Citation Analysis as an Unobtrusive Method for Journal Collection Evaluation Using Psychology Student Research Bibliographies", Collection Building, vol. 17, núm. 1, pp. 20-28.

Sylvia, M. y M. Lesher (1995), "What Journals Do Psychology Graduate Students Need?: a Citation Analysis of Thesis References", College and Research Libraries, vol. 56, núm. 4, p. 313.

Urbano Salido, C. (2001), "El análisis de citas en trabajos de investigadores como método para el estudio del uso de información en bibliotecas", Anales de Documentación, núm. 4, pp. 243-266.

Walcott, R. (1994), "Serials Cited by Marine Sciences Research Center Faculty, University at Stony Brook, 1986-1991”, Science and Technology Libraries, vol. 14, núm. 3, pp. 15-33. 